\title{
Avifauna en sistemas silvopastoriles en el Corredor Biológico Mesoamericano, Tabasco, México
}

\author{
Noel González-Valdivia ${ }^{1}$, Everardo Barba-Macías²*, Salvador Hernández-Daumás (qepd) \&
} Susana Ochoa-Gaona ${ }^{1}$

1. El Colegio de la Frontera Sur, Depto. de Ciencias de la Sustentabilidad. Adaptación Humana y Manejo de Recursos en Ecosistemas Tropicales, Unidad Campeche. Apdo. Postal No. 48, Lerma, Campeche, Campeche, México; siankaan2003@gmail.com, sochoa@ecosur.mx

2. El Colegio de la Frontera Sur, Depto. de Ciencias de la Sustentabilidad. Manejo sustentable de cuencas y zona costera. Unidad Villahermosa. Carretera Villahermosa-Reforma km. 15.5, Ranchería Guineo 2a sección C.P. 86280 Villahermosa, Tabasco, México; ebarba@ecosur.mx

* Correspondencia.

\section{Recibido 19-VIII-2013. Corregido 10-II-2014. Aceptado 11-III-2014.}

\begin{abstract}
Avifauna in silvopastoral systems in the Mesoamerican Biological Corridor, Tabasco, México. Silvopastoral systems support local ecological and economical features as they enhance conservation of floral and faunal communities. As other animal communities, avifauna may be a good representative of habitat alterations, both as the species and functional levels. In order to attend the initiative of Mesoamerican Biological Corridor initiative (CBM) in the state of Tabasco, we studied the diversity of birds in two silvopastoral systems: scattered trees in pastures (ADP), and trees in boundary-hedgerows (AL). For this, we applied the fixed radius counting point method in three priority sites in Tabasco's CBM during the dry and wet season of 2011, and a total of 56, 60 and 62 points were evaluated in Huimanguillo, Tenosique and Tacotalpa, respectively. We observed 2084 individuals of 154 species (79-89\% of expected diversity) and 36 bird families. We detected 92, 87 and 85 species in Huimanguillo, Tenosique and Tacotalpa, respectively, including 35 protected species, of which 23, 19 and 16 in each locality, respectively. All sites showed high diversity $\left(H^{\prime} \geq 3.20\right)$, low species dominance $(\mathrm{D} \geq 0.08)$ and high equitability $(\mathrm{J} \geq 0.77)$. Species composition showed differences between sites, being most similar Tacotalpa and Tenosique. Ten species were considered characteristic for sites. Although the silvopastoral system did contain protected species, the low diversity and the early successional character of the arboreal components were not attractive to frugivorous bird species. Diversification with native trees can improve the systems to create a complementary habitat and to increase landscape connectivity. The management of silvopastoral practices on cattle dominated landscapes in Tabasco could improve its ecological quality, and thus achieve the CBM's objectives of biodiversity conservation combined with human economic activities. Rev. Biol. Trop. 62 (3): 1031-1052. Epub 2014 September 01.
\end{abstract}

Key words: agricultural matrix, agroecosystems, connectivity, hedgerow trees, live fences, scattered trees in pastures, species diversity.

El establecimiento de mecanismos y diseño de estrategias que ayuden a la conservación de los recursos biológicos regionales se fortalece con el establecimiento del Corredor Biológico Mesoamericano (CBM), cuya meta es la conservación biológica mediante la conexión de áreas protegidas y otros remanentes de bosques (Domínguez-Cervantes, 2009). Esta conectividad se puede mejorar con acciones incluyentes y alternativas sustentables en los territorios (Independent Evaluation GroupWorld Bank, 2011). El CBM se impulsa en Panamá, Costa Rica, Nicaragua, Honduras, El Salvador, Guatemala, Belice y México (Comisión Centroamericana de Ambiente y Desarrollo, 2002). Específicamente, en el territorio mexicano la estrategia inició en los estados de Chiapas, Campeche, Quintana Roo y Yucatán 
(Ramírez, 2003) y recientemente, se ha promovido el establecimiento del CBM en el estado de Tabasco (Secretaría de Desarrollo Social y Protección al Ambiente, 2006), con el fin de facilitar la conexión biológica entre los remanentes de bosques tropicales, además de mejorar la conexión regional con los bosques de Veracruz, Chiapas y Campeche, en México y El Petén, Guatemala, Centroamérica.

En Tabasco, el bosque tropical lluvioso ocupa solamente $8 \%$ de su extensión original debido al avance de la agricultura, la ganadería y más recientemente de la industria extractiva de petróleo (Maldonado et al., 2008; RenteríaGarita, 2009). La deforestación ha afectado tanto la distribución espacial de los bosques nativos, como la diversidad biológica que albergan (Ehrlich, 1988; Myers, 1988; Masera, Ordóñez, \& Dirzo, 1997). Actualmente, al menos $70 \%$ de la superficie estatal esta dedicada a la ganadería extensiva (Ruiz-Rodríguez, 2000; Manjarrez et al., 2007), incluyendo la ocupada por humedales (Barba-Macías, Rangel-Mendoza, \& Ramos-Reyes, 2006). Los remanentes de bosque tropical se localizan particularmente en las sierras de Tenosique, Tacotalpa y Huimanguillo, con fragmentos menores dispersos en los lomeríos y planicies de la región Chontalpa (Secretaría de Desarrollo Social y Protección al Ambiente, 2006). Estos representan los últimos ecosistemas forestales en el Estado, por lo que urge su conservación y ampliación. De manera complementaria, mediante las prácticas de manejo que incorporan, intensiva o extensivamente, especies arbóreas nativas, la tradición y cultura agroforestal campesina ha impedido la pérdida total del componente arbóreo y de la diversidad biológica en el paisaje (Maldonado et al., 2008). Al respecto, Cabrera et al. (2012) mencionan que el conocimiento local de especies forestales de uso múltiple, además de amplio, resulta relevante en la mejora del manejo en las zonas agropecuarias de Tabasco.

Conectar los remanentes forestales puede ser posible mediante la promoción de los sistemas agroforestales tradicionales mejorados, para incluirlos en las estrategias de manejo del territorio (Harvey, Tucker, \& Estrada, 2004). Con ello se esperaría que la matriz agropecuaria se permeabilice al flujo biológico y se haga más apto para la fauna nativa. Si este efecto se demuestra mediante investigación, tales prácticas de manejo deberían integrarse ampliamente al CBM, como escenarios para impulsar una cultura más sustentable del uso de los recursos naturales (Gomiero, Pimentel, \& Paoletti, 2011). Así, resulta ecológicamente importante investigar los sistemas agroforestales en particular los de tipo silvopastoril (SP) de árboles dispersos o aislados en potreros (ADP) y árboles en cercos vivos o linderos (AL) (Sinclair, 1999), que son los más frecuentemente establecidos en Tabasco (Maldonado et al., 2008). Su aporte en la conservación de especies debe evaluarse, para determinar si este manejo campesino, puede ser incorporado en el manejo sustentable de la agricultura tropical (Gliessman, García, \& Amador, 1981). Es relevante conocer la biodiversidad asociada a estos agroecosistemas y determinar si funcionan efectivamente como hábitats alternativos o como corredores, en especial para especies propias de bosques.

Los sistemas agroforestales y su aporte ecológico o económico, han sido estudiados en Mesoamérica por distintos grupos de investigadores como Guevara \& Laborde (1993); Harvey \& Haber (1999); Jiménez et al. ( 2007); Harvey et al., (2008); Jiménez, Velasco, Uribe \& Soto (2008) y Pinto-Ruiz et al. (2010). En Tabasco, los trabajos de González et al. (2010); González et al. (2011); González et al. (2012a); González et al. (2012b); González et al. (2013) y Pérez, Van der Wal \& Ishiki (2012), evidencian la capacidad de la flora y fauna de persistir en los agroecosistemas. De acuerdo con Sans (2007), el aumento de la diversidad favorece la diferenciación de hábitat, incrementa las oportunidades de coexistencia y de interacción entre las especies, y generalmente lleva asociada una mayor eficiencia en el uso de los recursos. De manera general, los agroecosistemas más diversificados -que suelen coincidir con los gestionados mediante prácticas de la agricultura ecológica y tradicional- tienen 
mayores ventajas que los altamente simplificados; desde esa base, las estrategias de conservación biológica serán más eficaces dentro del CBM en Tabasco.

Dado que en términos prácticos no es posible conocer toda la diversidad biológica de un territorio, se puede recurrir a un grupo biológico representativo de la misma que funcione como indicador ecológico (Spellerberg, 2005). Las aves pueden ser útiles en este aspecto, debido a que se detectan e identifican con relativa facilidad en campo y son sensibles a las modificaciones de los hábitats, tanto a nivel de especie como de grupos funcionales (Arriaga, Calme, \& Kampichler, 2008). Tabasco es notable por su riqueza en avifauna (Winker, Arriaga, Trejo \& Escalante, 1999), se han registrado 490 especies de aves (Chablé, Escalante, \& López, 2005), de las cuales al menos 87 han sido observadas en SP de ADP y 76 en AL (Koller, Arriaga, \& Maldonado, 2010) (González et al., 2011).

El objetivo planteado fue analizar los cambios en la diversidad y composición de especies de aves que utilizan los sistemas silvopastoriles (ADP y AL) en tres sitios prioritarios para la conservación de la biodiversidad en Tabasco (CONABIO, 2009). La información es relevante en la definición de estrategias de implementación del CBM en Tabasco, porque considera las prácticas agroforestales realizadas por los campesinos de la región y porque permite establecer una línea base de comparación para monitoreo de la avifauna dentro de este territorio.

\section{MATERIALES Y MÉTODOS}

Zona de estudio: El estudio incluyó tres sitios dentro de los municipios de Huimanguillo, Tenosique y Tacotalpa, del estado de Tabasco, ubicados donde domina el bosque tropical húmedo, y seleccionados como áreas prioritarias para la conservación (Arriaga, Aguilar, \& Espinoza, 2000). Estos se seleccionaron por la presencia de los dos sistemas silvopastoriles dentro de ellas, en función del permiso de los lugareños para el ingreso a sus fincas, y por su cercanía a extensiones importantes de bosques tropicales lluviosos, que actúan como núcleos de conservación de biodiversidad por su proximidad a masas forestales presentes en los estados vecinos de Chiapas, Campeche y Veracruz, México y El Petén, Guatemala, Centroamérica.

El sitio de Huimanguillo corresponde a lomeríos bajos, de composición sedimentaria, caracterizado por un relieve ondulado de baja altitud $(<50 \mathrm{msnm})$ con dirección Este-Oeste, ubicado en la Provincia Fisiográfica de la Llanura Costera del Golfo Sur. Los sitios montañosos de Tacotalpa y Tenosique, presentan características del relieve cárstico, que alcanzan hasta los $800 \mathrm{msnm}$ y pertenecen a la Provincia de la Sierra de Chiapas y Guatemala. Con un clima cálido y húmedo en todos los sitios, con precipitaciones de $2000-4000 \mathrm{~mm}$ anuales y temperatura media anual superior a los $24^{\circ} \mathrm{C}$. La vegetación original para todos los sitios era el bosque tropical lluvioso, alto a mediano en Tenosique y Tacotalpa, pero predominantemente de tipo inundable a ribereño, con encinares de Quercus oleoides en las partes altas en Huimanguillo (Secretaría de Desarrollo Social y Protección al Ambiente, 2006).

El sitio de lomeríos bajos (Huimanguillo), se incluye como posible área de conexión para la fauna terrestre de los remanentes boscosos, pero también para aves y fauna acuática por contener un sistema lagunar importante, como la Laguna de Rosario, ubicado cerca de otros humedales marino-costeros y lagunares del Estado de Tabasco y del vecino Estado de Veracruz, México. Las dos localidades seleccionadas en este sitio, fueron Central Fournier $1^{a}$ Sección (17 $\left.52^{\prime} 02^{\prime \prime} \mathrm{N}-93^{\circ} 55^{\prime} 13^{\prime \prime} \mathrm{W}\right)$ y Central Fournier $2^{\mathrm{a}}$ Sección $\left(17^{\circ} 53^{\prime} 03^{\prime \prime}\right.$ N 935'13” W). En Tenosique, en el territorio conectado con la Reserva El Petén, Guatemala y con la Selva Lacandona, Chiapas, México, que además se relaciona con la Reserva de Biósfera Pantanos de Centla por conducto del bosque ribereño distribuido a lo largo del Rio Usumacinta, se incluyeron las comunidades de Ignacio Allende $\left(17^{\circ} 17^{\prime} 59^{\prime \prime} \mathrm{N}-91^{\circ} 13^{\prime} 08^{\prime}\right.$ ' W) y Santo Tomás $\left(17^{\circ} 18^{\prime} 16^{\prime \prime} \mathrm{N}-91^{\circ} 21^{\prime} 15^{\prime \prime} \mathrm{W}\right)$; mientras que en Tacotalpa, conectado con el 
CBM en su trayecto por las sierras de Chiapas, se incluyeron las comunidades de Zunú-Patastal $\left(17^{\circ} 28^{\prime} 21^{\prime \prime} \mathrm{N}-92^{\circ} 48^{\prime} 50^{\prime}\right.$ " W) y Villa Luz (17²6’29” N - 92 $45^{\circ} 56^{\prime}$ ” W).

Descripción de los sistemas silvopastoriles por zona: En Tabasco, cinco décadas de deforestación impulsada principalmente por la ganadería extensiva, que ocupa actualmente $70 \%$ del territorio, han dejado menos que $8 \%$ del bosque tropical original (Martínez, Villanueva \& Casanova, 2013) y las iniciativas para repoblar con árboles el Estado se hacen urgentes si se pretende conservar y restaurar la biodiversidad local. Dos sistemas agroforestales de tipo silvopastoril fueron seleccionados para este estudio: los ADP y los AL (Nair, 1985). Se caracterizaron estos sistemas usando variables estructurales y ambientales medidas en parcelas temporales de $5 \mathrm{~m} \times 50 \mathrm{~m}$ establecidas dentro de los sistemas seleccionados en cada sitio. En el caso de ADP, la parcela iniciaba en la posición de un árbol y se orientaba de Este a Oeste. En los AL se buscó ubicar la parcela centrada sobre la cerca o lindero que tuviese la mayor aproximación a la dirección Este-Oeste. Con esta medida se trató de disminuir sesgos y de estandarizar el método de establecimiento de parcelas de muestreo.

Las variables ambientales medidas fueron: temperatura $\left({ }^{\circ} \mathrm{C}\right)$ y humedad relativa $(\%)$ (Barómetro-Termo higrómetro Steiner) y altitud (m; Altímetro Casio Forester FTS-100). Las variables estructurales se basaron considerando árboles $\geq 5 \mathrm{~cm}$ de dap (diámetro medido a $1.30 \mathrm{~m}$ de altura en el fuste) a partir de lo cual se calculó: densidad de individuos (ind./ha), área basal $\left(\mathrm{m}^{2} / \mathrm{ha}\right)$, promedio del diámetro de copa y altura media del fuste de los árboles por SP.

Diseño de muestreo de aves: Mediante el apoyo de las autoridades de cada comunidad y con el permiso de los propietarios, se visitaron las fincas en las que se ubicaron los puntos de conteo. En las comunidades se ubicaron cuatro fincas o parcelas (excepto en Villa Luz, Tacotalpa con sólo dos) que contuviesen al menos uno de los sistemas de manejo seleccionados, fuese ADP o de AL. En total se trabajaron 21 fincas. Durante 2011 se establecieron 140 puntos de conteo con radio fijo $(25 \mathrm{~m})$, para el registro de la avifauna observada o escuchada dentro del perímetro (Bibby, Jones, \& Marsden, 1998). Un total de 73 puntos se establecieron en sistemas de ADP, con 27 distribuidos en Huimanguillo, 23 en Tenosique y 23 en Tacotalpa. Por otro lado, 67 puntos se ubicaron en sistemas de AL, con 26 en Huimanguillo, 17 en Tenosique y 24 en Tacotalpa, respectivamente. Todos los puntos fueron visitados una vez en la época seca (abril-junio) y otra en la lluviosa (julio-octubre). El cuadro 1 resume el muestreo realizado y las características ambientales de cada SP.

La observación y registro de aves fue diurna, entre las 06:00-09:30 horas. La distancia mínima entre los puntos fue de $150 \mathrm{~m}$, con observaciones durante diez minutos en cada punto, avanzando durante al menos cinco minutos a la siguiente posición. Las rutas se trazaron aleatoriamente, pero avanzando de manera que los puntos de conteo quedasen ubicados dentro de los límites de cada SP visitado en cada finca. En general, se alternaron los sistemas de ADP con los de AL. Con base en la detección directa o auditiva, realizadas por el mismo investigador, todos los individuos fueron registrados e identificados a nivel de especie con apoyo en guías de campo para aves de México y Centroamérica, así como para aves de Norteamérica (Howell \& Webb, 1995; Peterson \& Chalif, 2006; Van Perlo, 2006). Se siguió la nomenclatura propuesta por la American Ornithologists' Union (1998). Además se determinaron las especies consideradas en riesgo e incluidas dentro de las diferentes categorías de protección contenidas en la NOM-059-SEMARNAT (Diario Oficial de la Federación, 2010).

Las especies se agruparon, siguiendo la adaptación que hace González-Valdivia et al. (2012b) con base en Robinson (2001) y Gillespie (2002), en los gremios de forrajeo: cazador acuático (CA), carroñero (Car), cazador que busca presas perforando la madera (Cro), cazador asociado a ganado vacuno (Gan), 
CUADRO 1

Características ambientales en los sistemas silvopastoriles estudiados en el área de influencia del Corredor Biológico Mesoamericano en Tabasco

TABLE 1

Environmental characteristics in the studied silvopastoral systems in the influence area of Mesoamerican Biological Corridor in Tabasco, Mexico

\begin{tabular}{ccccccccccc} 
Zona & Sistema & \multicolumn{4}{c}{ Variables ambientales } & \multicolumn{5}{c}{ Variables estructurales } \\
& & HRS & HRL & TS & TL & Alt & C & AT & DA & AB \\
H & AL (26) & 75.1 & 86.3 & 27.1 & 25.5 & 9.5 & 4.5 & 2.6 & 450 & 24.7 \\
& ADP (27) & 75.3 & 86.0 & 26.3 & 25.6 & 9.1 & 5.7 & 3.2 & 169 & 12.0 \\
Ta & AL (24) & 74.3 & 77.7 & 26.2 & 26.6 & 167.7 & 4.1 & 2.7 & 870 & 9.0 \\
& ADP (23) & 73.9 & 77.3 & 27.0 & 27.1 & 167.4 & 6.5 & 2.9 & 187 & 4.2 \\
Te & AL (17) & 68.8 & 88.5 & 27.2 & 25.0 & 117.7 & 4.6 & 3.1 & 775 & 23.2 \\
& ADP (23) & 67.9 & 88.7 & 27.4 & 24.4 & 140.9 & 6.0 & 3.2 & 165 & 5.6 \\
\hline
\end{tabular}

H: Huimanguillo, Ta: Tacotalpa, Te: Tenosique. AL: árboles en linderos, ADP: árboles dispersos en potreros. Entre paréntesis número de puntos evaluados. Variables ambientales: HRS: humedad relativa en estación seca (\%), HRL: humedad relativa en estación lluviosa $(\%)$, TS: temperatura en estación seca $\left({ }^{\circ} \mathrm{C}\right)$, TL: temperatura en estación lluviosa $\left({ }^{\circ} \mathrm{C}\right)$, Alt: altitud $(\mathrm{msnm})$. Variables estructurales: C: diámetro promedio de la copa arbórea $(\mathrm{m})$, AT: altura media del tronco (m), DA: densidad de árboles (ind./ha), AB: área basal ( $\left.\mathrm{m}^{2} / \mathrm{ha}\right)$.

H: Huimanguillo, Ta: Tacotalpa, Te: Tenosique. AL: trees in boundary-hedgerows, ADP: scattered trees in pastures. Into brackets the number of evaluated points. Environmental variables: HRS: relative humidity in dry season (\%), HRL: relative humidity in rainy season $(\%)$, TS: dry season temperature $\left({ }^{\circ} \mathrm{C}\right)$, TL: rain season temperature $\left({ }^{\circ} \mathrm{C}\right)$, Alt: altitude (mals). Structural variables: C: mean tree-crown diameter (m), AT: stem mean height (m), DA: trees density (ind./ha), AB: basal area $\left(\mathrm{m}^{2} / \mathrm{ha}\right)$.

granívoro-frugívoro arbóreo (Gfa), granívoro-frugívoro terrestre (Gft), nectarívoro $(\mathrm{N})$, omnívoro arbóreo (OA), omnívoro $(\mathrm{OM})$, insectívoro con apariencia paseriforme sin percha para cazar del estrato arbustivo alto a medio (Pam), insectívoro con apariencia paseriforme sin percha para cazar del estrato arbustivo bajo a herbáceo (Pbh), rapaz diurna (RD), rapaz nocturna $(\mathrm{RN})$, insectívoro que caza en el suelo o en la hojarasca (S), insectívoro grande a mediano de apariencia tiraniforme que caza desde perchas bajo el dosel arbóreo (Tg), insectívoro pequeño de apariencia tiraniforme que caza desde perchas bajo el dosel arbóreo (Tp).

La eficacia de la detección de especies con base en el esfuerzo de muestreo, fue evaluada mediante la determinación del porcentaje de especies registradas respecto del total esperado (completitud de inventario en \%). Para esto se emplearon las curvas de rarefacción de Coleman y utilizando como valor esperado de especies al máximo valor de la serie obtenida para el índice Chao1, recomendado para estimaciones basadas en abundancia (Chao, Chazdon, Colwell, \& Tsung-Jen, 2006). En los cálculos de todos estos índices fue utilizado EstimateS 8.0 (Colwell, 2008).

Mediante el análisis de correlación de Pearson, se determinaron las relaciones entre la riqueza de especies, los gremios de forrajeo y el registro de individuos respecto a las variables estructurales y ambientales de los SP estudiados (Legendre, 2007). La diversidad de aves, en los sitios y en los SP anidados en ellos, fue medida a través de índices de diversidad de Shannon-Wiener, de dominancia de Simpson y de equidad de Pielou (Magurran, 1988; Moreno, 2001).

Utilizando los registros de la detección de especies por punto de conteo como un estimador de las abundancias, se comparó la composición comunitaria entre unidades a través de análisis de apareamiento de medias aritméticas no ponderadas (UPGMA), tomando el índice 
asimétrico de Bray-Curtis como medida de distancia (Zuur, Leno, \& Smith, 2007). Los cálculos multivariados de clasificación fueron realizados mediante la aplicación PAST 2.13 (Hammer, Harper, \& Ryan, 2001) y los de correlación mediante SPSS 10.0 (Morrison, 1999).

Las especies indicadoras fueron determinadas siguiendo el método IndVal propuesto por Dufrene \& Legendre (1997), utilizando PcOrd 4.25 (McCune \& Mefford, 1999). El IndVal determina la cualidad de una especie como indicadora de un ambiente particular basándose en dos criterios: la especificidad y la fidelidad. La especificidad se refiere a la frecuencia y la abundancia con que se presenta la especie en todos los ambientes (o condiciones ambientales) incluidos en el estudio. La fidelidad se refiere a que tanto la especie se presenta en un ambiente o condición en particular, lo caracteriza por ser exclusiva del mismo, por lo tanto funciona como indicadora del mismo.

\section{RESULTADOS}

Sistemas silvopastoriles: Las condiciones microclimáticas en los que se ubican los SP estudiados variaron entre sitios. La variable que más destacó por sus extremos fue la altitud, que en promedio fue más baja en Huimangui1lo, mientras que en Tacotalpa se registran las mayores altitudes promedio. En Tenosique, los SP se encontraron a una altura promedio cercana a las de Tacotalpa (Cuadro 1). La temperatura entre sitios fluctuó hasta en $1.5^{\circ} \mathrm{C}$, con variaciones entre estaciones. La humedad relativa varió tanto entre sitios como entre estaciones del año y en ambos casos, las diferencias entre los extremos se acercaron al 10\%.

El arbolado en los ADP, muestra que Tacotalpa presentó una mayor densidad que Huimanguillo y Tenosique (Cuadro 1). En Huimanguillo estuvo dominado por Miconia argentea (Sw.) DC. (Melastomataceae, 19\%), Eucalyptus sp. (Myrtaceae, 13\% especie introducida), Attalea cohune Mart. (Arecaceae, $12 \%$ ) y Byrsonima crassifolia (L.) Kunth (Malpighiaceae, 12\%). En Tacotalpa el arbolado estuvo representado por Tabebuia rosea (Bertol) A. DC. (18\%, Bignoniaceae), Tabebuia donnell-smithii Rose (Bignoniaceae, $12 \%$ ) y Lysiloma sp. (Fabaceae, 12\%) como especies frecuentes. En Tenosique, la especie más frecuente fue Cedrela odorata L. (Meliaceae, 28\%) seguida por Guazuma ulmifolia Lam. (Malvaceae, 16\%).

En los AL, Tacotalpa supera en densidad arbórea a Tenosique y Huimanguillo, en este mismo orden (Cuadro 1). En esta última zona, el arbolado tuvo como especies frecuentes a Miconia argentea (29\%), Jatropha curcas L. (Euphorbiaceae, 14\%) y Cupania dentata DC. (Sapindaceae, 13\%). En Tacotalpa la especie más frecuente fue Gliricidia sepium (Jacq.) Kunth ex. Walp. (Fabaceae, 88\%) en un arreglo que semeja un monocultivo arbóreo de esta especie, sujeto a podas frecuentes. La especie más próxima fue Cedrela odorata que apenas superó el 3\% en abundancia relativa. En Tenosique hubo dos especies co-dominantes Guazuma ulmifolia y Cupania dentata DC. (Sapindaceae), ambas con 17\%.

El área basal del arbolado disperso en potreros de Huimanguillo promedió $12 \mathrm{~m}^{2} / \mathrm{ha}$, en Tacotalpa $4.2 \mathrm{~m}^{2} / \mathrm{ha}$, y en Tenosique $5.6 \mathrm{~m}^{2} /$ ha. En los linderos, el arbolado resultó con mayores valores para el área basal y el más alto se observó en Huimanguillo $\left(24.7 \mathrm{~m}^{2} / \mathrm{ha}\right)$, seguido por Tenosique $\left(23.2 \mathrm{~m}^{2} / \mathrm{ha}\right)$, mientras Tacotalpa observó el menor promedio con $9 \mathrm{~m}^{2} /$ ha (Cuadro 1). Tanto la copa media del árbol como la altura media del fuste obtuvieron mayores valores en los ADP que en los AL, independientemente del sitio de estudio donde se ubicaran (Cuadro 1).

Diversidad de la avifauna asociada a los tres sitios prioritarios: Se registraron 2 084 individuos de 154 especies incluidas en 36 familias (Apéndice), que representan el $89 \%$ de las 172 especies esperadas en estos tipos de sistemas agropecuarios en Tabasco. Las comunidades de aves en todos los sitios fueron semejantes, con diferencias de siete especies entre la más rica (Huimanguillo) respecto a la menos rica (Tacotalpa). El esfuerzo de muestreo permitió una detección de al menos el 79\% de 
completitud de inventario de las especies esperadas en cada uno de los tres sitios (Cuadro 2).

La diversidad por sitio mostró valores similares en el índice Shannon-Wiener (Cuadro 2). Las especies de las comunidades estudiadas mostraron una distribución equitativa en sus abundancias, por lo que la dominancia de especies fue uniformemente baja (Simpson, $\mathrm{D}<0.06$ ), mientras que la equidad tiende a ser alta (Pielou, $\mathrm{J}>0.76$ ). No obstante, la composición de las comunidades de aves cambió entre los sitios. El análisis de clasificación distingue tres grupos con las comunidades de aves de los sitios serranos en Tacotalpa y Tenosique más próximos entre sí (Similitud Bray-Curtis $>0.45$ ) y separados de la comunidad de aves de los sitios de lomeríos bajos estudiada en Huimanguillo (Similitud Bray-Curtis<0.40; Fig. 1).

\section{Análisis de la detección de aves en escala} temporal por sitio prioritario: No se encontraron diferencias significativas en la detección de especies, ni entre sitios (ANOVA $\mathrm{F}_{2}=0.973$, $\mathrm{p}=0.385$ ) ni entre estaciones del año (ANOVA $\mathrm{F}_{1}=2.82, \mathrm{p}=0.065$ ), con la menor detección de especies por punto registrada en Tacotalpa durante la estación lluviosa. La cantidad de individuos registrados entre sitios y estaciones se modifica significativamente tanto entre sitios (ANOVA $\left.\mathrm{F}_{2}=5.62, \mathrm{p}=0.018\right)$ como entre estaciones (ANOVA $\mathrm{F}_{1}=10.91, \mathrm{p}=0.001$ ).

Cuando se evalúa el registro de la avifauna en los SP por estación y sitio, los resultados muestran que durante la época seca se registran hasta cinco especies por punto en todas las localidades. Durante la época lluviosa el mayor registro de especies por punto de conteo lo presentaron igualmente Tenosique y Huimanguillo con cuatro y en Tacotalpa solo se registraron dos (Cuadro 3). Por otro lado, considerando la cantidad total de registros obtenidos en todo el periodo, se observa que en Huimanguillo y Tacotalpa se contabilizaron en promedio siete individuos por punto de conteo, similar al promedio en Tenosique con ocho individuos. El patrón observado es que en Tenosique se incrementa la detección en la estación lluviosa, mientras que en Tacotalpa disminuye, quedando Huimanguillo con resultados mixtos para este aspecto del monitoreo.

\section{Relaciones entre avifauna y las carac-} terísticas estructurales y ambientales: $\mathrm{La}$ densidad de árboles (ind/ha) mostró una correlación inversa con la cantidad de registros de individuos de aves detectados (Pearson, $\mathrm{r}=-0.24, \mathrm{p}=0.005)$ y con la cantidad de especies (Pearson, $r=-0.17, p=0.043$ ). Ninguna otra

CUADRO 2

Índices de diversidad de la avifauna para los sistemas silvopastoriles en Tabasco, México

TABLE 2

Sites diversity indexes of the avifauna for silvopastoral systems in Tabasco, Mexico

\begin{tabular}{|c|c|c|c|}
\hline \multirow{2}{*}{ Índices } & \multicolumn{3}{|c|}{ Municipio } \\
\hline & Huimanguillo & Tacotalpa & Tenosique \\
\hline Puntos de conteo & 53 & 47 & 40 \\
\hline Especies observadas & 92 & 85 & 87 \\
\hline Especies esperadas & 106 & 117 & 97 \\
\hline Completitud del inventario \% & 87 & 73 & 90 \\
\hline No. de especies amenazadas & 23 & 19 & 16 \\
\hline Individuos registrados & 861 & 636 & 587 \\
\hline No. de Familias & 32 & 27 & 25 \\
\hline Shannon-Wiener H' & 3.70 & 3.56 & 3.66 \\
\hline Simpson D & 0.04 & 0.06 & 0.04 \\
\hline Equidad Pielou J & 0.82 & 0.79 & 0.82 \\
\hline
\end{tabular}


CUADRO 3

Diversidad, riqueza e índices ecológicos de los sistemas silvopastoriles estudiados en Tabasco, México

TABLE 3

Diversity, richness and ecological indexes of the studied silvopastoral systems in Tabasco, Mexico

\begin{tabular}{lccccccccccc} 
& SP & R & Ind & Di & Do & E & ES & ELL & Ind/S & Ind/LL & Prot \\
Huimanguillo & AL & 62 & 346 & 3.34 & 0.03 & 0.86 & $4 \pm 3$ & $3 \pm 2$ & $7 \pm 4$ & $7 \pm 6$ & $15(67)$ \\
& ADP & 70 & 515 & 3.51 & 0.05 & 0.83 & $5 \pm 2$ & $4 \pm 2$ & $9 \pm 6$ & $11 \pm 9$ & $18(105)$ \\
Tacotalpa & AL & 64 & 258 & 3.51 & 0.05 & 0.84 & $4 \pm 2$ & $2 \pm 1$ & $7 \pm 4$ & $5 \pm 4$ & $12(82)$ \\
& ADP & 64 & 378 & 3.20 & 0.08 & 0.77 & $5 \pm 2$ & $2 \pm 1$ & $10 \pm 8$ & $7 \pm 6$ & $17(134)$ \\
Tenosique & AL & 53 & 218 & 3.45 & 0.05 & 0.87 & $5 \pm 2$ & $3 \pm 2$ & $7 \pm 5$ & $6 \pm 5$ & $9(17)$ \\
& ADP & 66 & 369 & 3.46 & 0.05 & 0.83 & $4 \pm 2$ & $4 \pm 2$ & $7 \pm 4$ & $10 \pm 7$ & $11(85)$ \\
\hline
\end{tabular}

SP: sistema silvopastoril, AL: árboles en linderos, ADP: árboles dispersos en potreros. R: riqueza: número de especies, Ind: total de individuos registrados, Di: Diversidad (índice de Shannon-Wiener), Do: Dominancia (índice de Simpson). E: Equitatividad (índice J de Pielou). ES: número de especies en secas, ELL: número de especies en lluvias. Ind./S: individuos registrados por punto en secas. Ind./LL: individuos registrados por punto en lluvias. Prot: número de especies listadas en la NOM-059-Semarnat (Diario Oficial de la Federación 2010) y en CITES (PNUMA-CMCM 2011), con el número de individuos registrado entre paréntesis.

SP: silvopastoral system, AL: trees in boundary-hedgerows, ADP: scattered trees in pastures. R: number of species, Ind: total of registered individuals, Di: diversity (Shannon-Wiener index). Do: dominance (Simpson index). E: equitability (Pielou $\mathrm{J}$ index). ES: number of species in dry season, ELL: number of species in rain season. Ind./S: all individuals registered by counting points in dry season, Ind./LL: all individuals registered by counting points in rain season. Prot: number of species listed in the NOM-059-Semarnat (Diario Oficial de la Federación 2010) and CITES list (PNUMA-CMCM 2011) with recorded individuals in parenthesis.
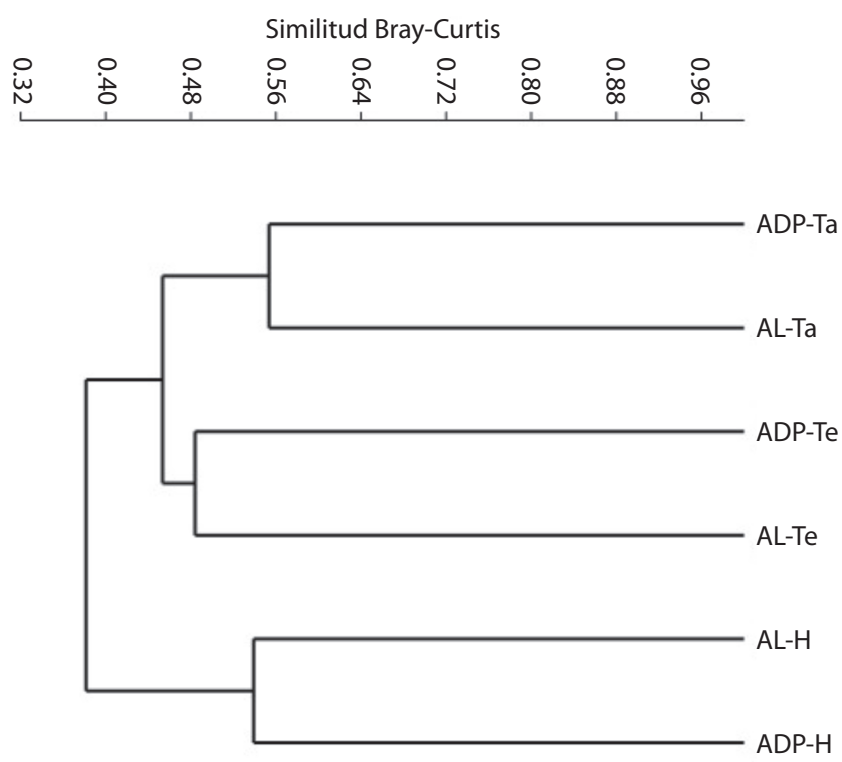

Fig. 1. Agrupamiento de las comunidades de aves en los tres sitios estudiados y los dos sistemas silvopastoriles de cada sitio. Sistemas silvopastoriles: ADP: árboles dispersos en potreros, AL: árboles en linderos. Sitios: H: Huimanguillo, Ta: Tacotalpa, Te: Tenosique.

Fig. 1. Birds' communities groups in the three studied sites and two silvopastoral systems in each one. Silvopastoral systems: ADP: scattered trees in pastures, AL: trees in boundary-hedgerows. Sites: H: Huimanguillo, Ta: Tacotalpa, Te: Tenosique. 
variable estructural ni ambiental presentó relaciones significativas respecto a los individuos registrados o la cantidad de especies de aves.

La riqueza de especies de aves por sistema silvopastoril no tuvo relaciones significativas con ninguna variable ambiental ni estructural $(\mathrm{p}>0.05)$. No obstante, desde un análisis autoecológico, 22 especies de aves presentaron relaciones con la altitud, mientras que ocho se vincularon con la humedad en periodo lluvioso y otras siete especies de aves se relacionaron con el área basal. La temperatura y el diámetro medio de copa se relacionaron con nueve y tres especies respectivamente (Apéndice).

De los 16 gremios forrajeros de aves identificados, seis mostraron relaciones significativas respecto a la altitud. Cinco de ellos, los cazadores acuáticos (CA), los cazadores que buscan presas perforando la madera (Cro), los granívoro-frugívoros terrestres (Gft), las rapaces diurnas $(\mathrm{RD})$ y los insectívoros grandes a medianos de apariencia tiraniforme que cazan desde perchas bajo el dosel arbóreo (Tg) correlacionan negativamente con esa variable ambiental (Pearson, $\mathrm{r}=-0.18$ a $0.29, \mathrm{p}<0.047$ ); mientras que el gremio de los insectívoros con apariencia paseriforme sin percha del estrato arbustivo bajo a herbáceo ( $\mathrm{Pbh}$ ) se relacionan positivamente con esta variable (Pearson, $\mathrm{r}=0.21, \mathrm{p}<0.015)$.

La humedad relativa en la temporada de lluvias mostró una relación directa con tres gremios forrajeros, Gft (Pearson, $\mathrm{r}=0.19$, $\mathrm{p}=0.028)$, Cro $(\mathrm{r}=0.18, \mathrm{p}=0.031)$ y $\mathrm{Tg}(\mathrm{r}=0.38$, $\mathrm{p}<0.001)$. La avifauna del gremio Tg se relacionó directamente con el área basal $\left(\mathrm{m}^{2} / \mathrm{ha}\right)$ y con la temperatura en temporada lluviosa $(r=0.32, p<0.001)$.

Se encontró que el 38\% de los grupos funcionales (6 de 16) presentaron alguna correlación con la altitud, mientras que al analizar las especies individuales sólo el $15 \%$ de las aves (22 de 154) mostró correlación con esa misma variable. La agrupación funcional proporcionó mejores respuestas sobre la relación de los organismos respecto a las condiciones del ambiente en que se encuentran.
Especies indicadoras y protegidas por sitio: Diez especies pueden ser consideradas como características (indicadoras) y distintivas para los sitios que se analizaron en Tabasco (IndVal $>0.7, p>0.05$ ). Estas fueron: Patagioenas flavirostris Wagler (Columbidae), Dendrocygna autumnalis Linnaeus (Anatidae), Sturnella magna Linnaeus (Icteridae) y Tyrannus melancholicus Cabanis (Tyrannidae) fueron propias de los lomeríos bajos de Huimanguillo. Setophaga fusca Muller (Parulidae) define los sitios de Tacotalpa, mientras, Contopus sordidulus Sclater, Myiozetetes similis Spix (Tyrannidae), Piranga rubra Linnaeus (Cardinalidae), Setophaga ruticilla Linnaeus (Parulidae) y Saltator atriceps Lesson (Incertae sedis), caracterizaron a Tenosique así como a la avifauna de una parte de la Sierra de Tacotalpa.

Fueron registradas un total de 23 especies incluidas en la norma de protección de vida silvestre vigente en México (Diario Oficial de la Federación 2010). De ellas, 14 se registraron en los SP en Huimanguillo, 12 en Tacotalpa y ocho en Tenosique. Las especies identificadas en alguna categoría de protección en México fueron: a) amenazadas: Botaurus lentiginosus Rackett (Ardeidae), Aramus guarauna Linnaeus (Aramidae), Aratinga holochlora Sclater, Pionus senilis Spix (Psittacidae), Pseudoscops clamator (Strigidae), Ramphastos sulfuratus Lesson (Ramphastidae) y Myadestes unicolor Sclater (Turdidae); b) en peligro de extinción: Cairina moschata Linnaeus (Anatidae) Amazona farinosa Boddaert y Amazona oratrix Ridgway (Psittacidae); y c) en la categoría de protección especial: Busarellus nigricollis Latham, Chondrohierax uncinatus Temminck, Ictinia plumbea Gmelin (Accipitridae), Falco femoralis Temminck (Falconidae), Patagioenas nigrirostris Sclater (Columbidae), Amazona albifrons Sparrman (Psittacidae), Pteroglossus torquatus Gmelin (Ramphastidae), Automolus ochrolaemus Tschudi (Furnariidae), Polioptila plumbea Gmelin (Polioptilidae), Catharus mexicanus Bonaparte (Turdidae), Euphonia gouldi Sclater (Thraupidae), Icterus spurius Linnaeus y Psarocolius montezuma Lesson (Icteridae). Por otra parte, un total de 
24 especies enlistadas en el convenio CITES (PNUMA-CMCM, 2011) fueron registradas en los SP dentro del CBM en Tabasco (Apéndice). De ellas, un total de 17 especies fueron registradas en los SP de Huimanguillo, mientras 12 especies se presentaron en Tacotalpa y en Tenosique, respectivamente. Entre las especies protegidas por la norma mexicana y aquellas enlistadas por el CITES, se suman en total 35 especies en peligro, que utilizan los SP en Tabasco, con 23 presentes en Huimanguillo, 19 en Tacotalpa y 16 en Tenosique, respectivamente (Apéndice).

Diversidad por sistema silvopastoril: La riqueza de aves en los sistemas de ADP de Huimanguillo, Tacotalpa y Tenosique alcanzó las 70, 64 y 66 especies, respectivamente $(84$, 79 y $86 \%$ de las esperadas en cada caso) con registros, en el mismo orden para cada sitio, de 515,378 y 369 individuos. Para el SP de AL se encontraron 62, 64 y 53 especies en Huimanguillo, Tacotalpa y Tenosique, respectivamente, detectando el 91,75 y $93 \%$ de las especies esperadas con 346, 258 y 218 individuos.

La diversidad alcanza valores altos en todos los SP (Shannon-Wiener, $\mathrm{H}^{\prime} \geq 3.20$ ), valores bajos para la dominancia de especies (Simpson, $\mathrm{D} \leq 0.08$ ), y altos para la equidad en la distribución de las poblaciones (Pielou, $\mathrm{J} \geq 0.77$, Cuadro 2).

El análisis de clasificación permitió distinguir tres grupos en función de los sitios estudiados. En Huimanguillo la similitud entre ambos SP se ubica en un 54\% y en Tacotalpa llega al $56 \%$. En Tenosique, ambos agroecosistemas se asemejan en cerca del $50 \%$. No se encontraron especies indicadoras para ninguno de los SP incluidos en esta investigación.

Análisis de la detección de aves en escala temporal por sistema silvopastoril: Respecto a la observación de aves en cada SP se observó que en la estación seca los registros promedio de especies por punto de conteo fueron entre cuatro a cinco mientras en la lluviosa desciende entre dos y cuatro especies (Cuadro 3). El número de individuos registrados por punto de conteo durante la época seca promedió entre siete y 10. El mismo dato en la estación lluviosa obtuvo un registro medio de cinco a 11 por punto.

Especies indicadoras $\mathbf{y}$ protegidas por sistema silvopastoril: Se encontraron especies protegidas en todos los SP establecidos en los distintos sitios. En los sistemas de ADP se tiene un registro de 11,17 y 18 especies listadas en la NOM-059-Semarnat y por el CITES para Tenosique, Tacotalpa y Huimanguillo respectivamente (Apéndice). Por otro lado, los sistemas de AL albergaron nueve especies en Tenosique, 12 en Tacotalpa y 15 especies protegidas en Huimanguillo (Cuadro 3).

\section{DISCUSIÓN}

Las correlaciones entre la riqueza de especies con las características ambientales y estructurales en los SP incluidos en el estudio se presentan más claramente cuando se analizan con respecto a los gremios de forrajeo, siendo la altitud y humedad relativa, tanto en la estación seca como en la lluviosa, las que tuvieron mayor influencia, siendo similar a lo encontrado por Chan-Dzul (2010) y GonzálezValdivia et al. (2012b).

La riqueza total de aves registradas en los SP visitados representa cerca del 32\% de la avifauna total reportada por Chablé-Santos et al. (2005) y es mayor del $50 \%$ al registro de especies reportado en diferentes SP de este estado (Van der Wal, Peña, Arriaga, \& Hernández, 2012). El registro de aves en ADP y en $\mathrm{AL}$, tanto en riqueza de especies como en el número de individuos en los SP en Tabasco coinciden con lo reportado por Cárdenas, Harvey, Ibrahim, \& Finegan (2003) y por Cerezo, Chandler, \& Dowell (2009) en Centroamérica, señalando que las aves observadas en ambientes silvopastoriles prefieren hábitats abiertos. Estrada, Estrada, \& Meritts (1997) y Estrada, Cammarano, \& Estrada (2000) también reportan resultados similares de riqueza de especies de aves en cercas vivas. 
Los SP estudiados presentan especies arbóreas dispersas, sucesionalmente tempranas o intermedias, con porte bajo, poco ramificadas y que no producen frutos carnosos. Por estas características, no resultan atractivos para especies que demandan una dieta frugívora, por ejemplo los crácidos grandes (Brooks \& Fuller, 2006), lo cual puede explicar la ausencia de aves representativas de interior de bosque en este estudio, siendo esto un inconveniente a solucionar en estos ambientes agropecuarios. A partir de estos resultados, es importante sugerir que los SP dentro del CBM en Tabasco deben establecerse en común acuerdo con los propietarios, para que a la vez que les proporcionen productos maderables y servicios, les permitan también cumplir funciones ecológicas. En Huimanguillo, un ejemplo de valor ecológico de una especie arbórea para una especie de ave en peligro de extinción, es el caso de la palma Attalea cohune, la cual favorece el anidamiento de Amazona oratrix (Psittacidae), en los potreros.

Muchas especies de árboles nativos multiusos han sido documentadas para Tabasco (Ochoa-Gaona, Pérez, \& Jiménez, 2008) y (Ochoa-Gaona et al., 2012). Su inclusión dentro de los sistemas silvopastoriles puede influir en un incremento de su calidad como hábitats y atraer una avifauna más diversa. Esto lograría un efecto de diversificación de nichos, generación de refugios o de espacios de reproducción, similar al efecto en la avifauna debido al incremento en complejidad estructural y la modificación de la composición arbórea durante la sucesión acontecida en el descanso agrícola (Shankar, Rawat, \& Johnsingh,1998).

La riqueza de aves resultó similar a los valores reportados por Arriaga-Weiss et al. (2008) y González-Valdivia et al. (2012b) en Tabasco y Estrada et al. (2000) en Veracruz. Esto nos permite validar que un estudio con un número menor de puntos ( $>20$ por sitio) como se hizo en este estudio, muestra resultados similares, lo cual es interesante en la planificación de estudios y monitoreo futuros de la avifauna en los corredores ecológicos en el sureste mexicano.
El índice de Shannon-Wiener, medido dentro de cada SP y en función de los sitios resultó muy alta $\left(\mathrm{H}^{\prime}>2.1\right.$ y $\mathrm{H}^{\prime}>3.3$; Ramírez-González, 2006), siendo mayores a los reportados por Cárdenas et al. (2003) en la provincia de Guanacaste, Costa Rica. La equidad para cada SP indica una tendencia al equilibrio entre las poblaciones de aves que cohabitan en cada uno de estos ambientes, lo que implica que las comunidades de aves en los SP de Tabasco podrían tener una adaptación a estos entornos, los cuales funcionan como hábitats que permiten su supervivencia. Esta situación resulta similar a la reportada para ambos tipos de SP por González-Valdivia et al. (2012b), y mayores a los reportados por Cárdenas et al. (2003) y Van der Wal et al. (2012).

La información generada debe ser considerada con mucho cuidado para evitar generalizaciones sesgadas ya que el valor de la diversidad por sí solo no es un parámetro para decidir la calidad de los hábitats estudiados y debe asociarse con otros, por ejemplo, la composición de las especies y la tipología de las mismas (Cerezo et al., 2009), siguiendo distintos criterios (especialización, grupo funcional, estado de vulnerabilidad o de riesgo, entre otros). En este sentido, la composición de las especies, coincidiendo con Cárdenas et al. (2003), Arriaga-Weiss et al. (2008) y Van der Wal et al. (2012), incluye principalmente a especies generalistas. Esta tendencia se ha encontrado dominantemente en Mesoamérica y ha sido detectada en estudios realizados sobre distintos SP (Harvey \& Haber 1999; Harvey et al., 2004; Harvey et al., 2005; Harvey et al., 2006; Harvey \& Villalobos, 2007; Cerezo et al., 2009). Por lo que es necesario diseñar estrategias que incluyan hábitats para especies del interior del bosque o más especializadas.

Cada SP en los sitios de estudio en Tabasco contiene una proporción de especies de aves diferente, que representó el efecto aditivo de las diferencias de ubicación geográfica (especialmente altitud) y del manejo que cada propietario hace de su finca. Así la mayoría de especies identificadas en Tenosique y Tacotalpa prefieren los bosques húmedos serranos, 
mientras que las identificadas en Huimanguillo se asocian a los bosques de zonas bajas inundables o humedales (Chablé-Santos et al., 2005). La distribución geográfica de los SP influye en la semejanza entre la diversidad y composición de especies de aves, con similitudes mayores entre las comunidades ubicadas en sistemas más próximos entre sí (Gillespie, 2002). Como mencionan Harvey et al. (2004, 2005, 2006), el cambio en las comunidades de aves también es expresión del disturbio, una respuesta al cambio en estructura y composición de la vegetación forestal ocasionado por el manejo silvopastoril en las floras locales, a lo largo del gradiente fisiográfico (Phillips, Hall, Gentry, Sawyer, \& Vázquez, 1994).

No obstante que el componente arbóreo en los SP permite la presencia de una tercera parte de la avifauna de Tabasco, las diferencias en la composición arbórea resultantes del manejo, parecen influir, a su vez, en la composición de la avifauna facilitando o inhibiendo la presencia de unas u otras especies o grupos funcionales. (Guevara \& Laborde 1993; Gillespie \& Walter 2001; Harvey \& González-Villalobos, 2007).

La amplitud del área de estudio, permite el registro de una proporción importante de la avifauna en el Tabasco. No obstante, en todos los casos se debe considerar que la heterogeneidad de la matriz agropecuaria actual, por si sola, no permite sostener a las restantes especies identificadas en el estado (aproximadamente 300). Es imprescindible la permanencia de áreas boscosas nativas dentro de los paisajes tabasqueños como única manera de sostener adecuadamente la diversidad de este grupo, en particular de los especialistas de interior de bosque (Daily, Ehrlich, \& Azofeifa, 2001; Cerezo et al., 2009; González-Valdivia et al., 2012b). Es importante la comparación de estos resultados con sus respectivos referentes ecológicos (bosques originales).

A pesar de que la densidad de árboles reportada por hectárea es alta, las especies dominantes (Cedrela odorata, Gliricidia sepium, Lysiloma spp., Miconia argentea) no resultan atractivas para las aves especialistas de bosque, como los nectarívoros y frugívoros
(Cárdenas et al. 2003), siendo estos SP catalogados como de baja calidad para la provisión de alimentos a las aves (Maridinou, Finegan, Jiménez, Delgado, \& Casanoves, 2013). En Tabasco, amplias superficies del territorio presentan muy baja calidad ecológica, ya que en el mejor de los casos estan cubiertas por potreros con árboles dispersos o en los linderos, con poca diversidad y pertenecientes a especies secundarias, por lo cual no están funcionando de la mejor manera como conexión entre parches de bosques remanentes en el territorio a nivel estatal o regional, limitando sus probabilidades de mantener un estado de conservación aceptable. Esta situación debe cambiarse mediante el enriquecimiento en la flora de los sistemas agropecuarios, de común acuerdo con los propietarios de parcelas y ranchos, que ayude a mitigar el impacto de la deforestación, considerado el principal problema en la región sureste de México (Ochoa-Gaona \& GonzálezEspinosa 2000; Manjarrez-Muñoz et al., 2007) y que afecta, además, otras áreas incluidas en el CBM en los estados de la región (Díaz, JeanFracois, \& Velázquez-Montes, 2008). Es por ello que no se detectan especies características, y la mayor parte de las aves son generalistas o especializadas a los hábitat abiertos (ArriagaWeiss et al., 2008; Van der Wal et al., 2012). Los grupos de especies que poseen mayor especialización al hábitat del interior del bosque lluvioso resultan los más afectados por los cambios del uso del suelo (Daily et al., 2001). En particular, lo anterior es debido a la intensa trasformación de los ecosistemas forestales a pastizales para la ganadería extensiva (Toledo, Batis, Becerra, Martínez, \& Ramos, 1995). La mayoría de las especies mencionadas en este trabajo han sido previamente documentadas en otros SP de la región mesoamericana (Cárdenas et al. 2003; Harvey et al. 2004; Cerezo et al., 2009; Van der Wal et al., 2012) y en su conjunto pueden considerarse como especies frecuentes, adaptadas a hábitats modificados por influencia humana o natural. Sin embargo, la presencia de especies en algún estado de conservación de acuerdo con la normatividad vigente (Diario Oficial de la Federación, 2010) 
o en la lista de especies CITES (PNUMACMCM, 2011), es un hecho importante, pues significa que los SP aún les sirven de cobertura. Por tanto, aún es posible recuperar, al menos parcialmente, la calidad ecológica de extensas superficies de hábitats degradados por la ganadería y agricultura en Tabasco en particular y en las áreas de corredor biológico, si se diversifica el número de especies arbóreas y se mejora su composición.

Considerando que los SP estan permitiendo que una parte de las especies de avifauna permanezca y se disperse a través del paisaje, se puede afirmar que mejorando la diversidad y composición de especies arborea los SP, pueden servir para mejorar la conectividad entre los remanentes forestales del territorio (Ferguson, Vandermeer, Morales, \& Daniel, 2003; Ferguson \& Griffith, 2004). Este manejo consiste en diseños en una perspectiva ecológica, con enriquecimiento de diversidad, utilizando especies arbóreas nativas y que provean de bienes y servicios al hombre, pero que a la vez puedan transformarse en hábitats aptos como corredores biológicos para las especies más exigentes o sensibles de la avifauna en Tabasco. Lo anterior incrementaría la capacidad de conservar los recursos avifaunísticos en las áreas prioritarias del CBM en Tabasco y en las regiones aledañas como son Veracruz, Chiapas, Campeche en México y El Petén en Guatemala. En ese sentido, se deben trabajar en la mejora de las prácticas agroforestales en el Estado de Tabasco con el objetivo de incrementar su calidad ecológica como hábitats para la avifauna local. Para esto resulta recomendable la consideración y aplicación apropiada de los conocimientos generados tanto por investigaciones realizadas en el territorio como el conocimiento tradicional de los campesinos y ganaderos.

\section{AGRADECIMIENTOS}

A FOMIX CONACYT-Tabasco por su apoyo mediante el proyecto "Aplicación del conocimiento agroforestal a la estrategia de implementación del Corredor Biológico Mesoamericano en Tabasco", TAB-2007-C09-74820.
A ECOSUR por su apoyo en infraestructura. A UCATSE por autorizar laboralmente la permanencia del primer autor en México. A los ejidatarios de Huimanguillo, Tenosique y Tacotalpa que dieron acceso a sus parcelas. A los revisores anónimos que ayudaron a mejorar este documento.

\section{RESUMEN}

Avifauna en sistemas silvopastoriles en el Corredor Biológico Mesoamericano, Tabasco, Mexico. Los sistemas silvopastoriles contienen rasgos ecológicos y económicos que contribuyen con la conservación de comunidades florísticas y faunísticas que en ellas se desarrollan. Entre otras comunidades faunísticas se encuentra la avifauna la cual es un grupo representativo de las alteraciones del habitat, tanto a nivel específico como grupo functional. Con el objetivo de atender la iniciativa de Corredor Biológico Mesoamericano en Tabasco, México, se analizó la diversidad de aves en dos sistemas silvopastoriles: árboles dispersos en potreros (ADP) y árboles en cercos o linderos (AL). Se aplicó el método de punto de conteo de radio fijo en tres sitios prioritarios del CBM de Tabasco, ubicados en los municipios: Huimanguillo, Tacotalpa y Tenosique. Los datos provienen de las estaciones seca y húmeda del 2011. Se registraron 2084 aves de 154 especies (79-89\% del esperado) y 36 familias. Se detectaron 92,87 y 85 especies, incluyendo 35 protegidas, con 23, 19 y 16 en Huimanguillo, Tacotalpa y Tenosique, respectivamente. Todos los sitios mostraron alta diversidad $\left(H^{\prime} \geq 3.20\right)$, baja dominancia de especies $(\mathrm{D} \geq 0.08)$ y alta equidad $(\mathrm{J} \geq 0.77)$. La composición de especies mostró diferencias entre sitios, con mayor similitud entre Tacotalpa y Tenosique. Diez especies pueden considerarse características de los sitios. Aunque en los sistemas silvopastoriles se refugian aves protegidas, el componente arbóreo es poco diverso y corresponde a especies de sucesión secundaria temprana, lo que limita a las aves frugívoras y especialistas de bosque. Diversificar estos sistemas con árboles nativos puede mejorar estos hábitats complementarios e incrementar la conectividad del paisaje para cumplir con los objetivos del CBM en la conservación de la biodiversidad y provisión de bienes a las poblaciones humanas.

Palabras clave: agroecosistemas, árboles dispersos en potreros, árboles en linderos o cercas vivas, diversidad de especies, matriz agropecuaria.

\section{REFERENCIAS}

American Ornithologists' Union. (1998). Check-list of North American Birds. The species of birds of North America from the Arctic through Panama, Including 
the West Indies and Hawaiian Islands. Washington, D.C., USA: American Ornithologists' Union.

Arriaga, L., Espinosa, J. M., Aguilar, C., Martínez, E., Gómez, L., \& Loa, E. (2000). Regiones terrestres prioritarias de México. DF, México: Comisión Nacional para el Conocimiento y Uso de la Biodiversidad. México.

Arriaga-Weiss, S. L., Calmé, S., \& Kampichler, C. (2008). Bird communities in rainforest fragment: guild responses to habitat variables in Tabasco, Mexico. Biodiversity and Conservation, 17, 173-190.

Barba, E., Rangel-Mendoza, J., \& Reyes, R. (2006). Clasificación de los humedales de Tabasco mediante sistemas de información geográfica. Universidad y Ciencia, 22(2), 101-110.

Bibby, C., Jones, M., \& Marsden, S. (1998). Expedition field techniques: bird surveys. Londres, Inglaterra: Expedition Advisory Centre, Royal Geographical Society.

Brooks, D. M. \& Fuller, R. A. (2006). Biología y conservación de crácidos (pp. 11-26). In L. Cancino \& D. M. Brooks (Eds.), Conservando Crácidos: la familia de aves más amenazada en Las Américas. Capítulo 1. Miscellaneous Publications of the Houston Museum of Natural Sciences, No. 6, Houston, Texas, USA.

Cabrera-Pérez, S., Ochoa-Gaona, S., Mariaca-Méndez, R., González-Valdivia, N., Guadarrama-Olivera, M. A., \& Gama, L. (2012). Vulnerabilidad por aprovechamiento y distribución de especies leñosas desde la perspectiva comunitaria en la reserva del Cañón del Usumacinta, Tenosique, Tabasco, México. Polibotánica, 35, 143-172.

Cárdenas, G., Harvey, C. A., Ibrahim, M., \& Finegan, B. (2003). Diversidad y riqueza de aves en diferentes hábitats de un paisaje fragmentado en Cañas, Costa Rica. Agroforestería en las Américas, 10 (39-40), 78-85.

Comisión Centroamericana de Ambiente y Desarrollo. (2002). El Corredor Biológico Mesoamericano: una plataforma para el desarrollo sostenible regional. Managua, Nicaragua: Corredor Biológico Mesoamericano-Comisión Centroamericana de Ambiente y Desarrollo. (Serie Técnica 1).

Cerezo, A., Robbins, C. S., \& Dowell, B. (2009). Uso de hábitat modificados por aves dependientes del bosque tropical en la región caribeña de Guatemala. Revista de Biología Tropical, 57(1-2), 401-419.

Chablé-Santos, J. B., Escalante-Pliego, P., \& LópezSantiago, G. (2005). Aves (p. 261-282). In J. Bueno, J. Álvarez, \& S. Santiago (Eds.), Biodiversidad del Estado de Tabasco. Capítulo 12. México, DF., México: Comisión Nacional para el Conocimiento y Uso de Biodiversidad (CONABIO) e Instituto de Biología de la Universidad Nacional Autónoma de México (IBUNAM).
Chan-Dzul, A. M. (2010). Diversidad florística y funcional a través de una cronosecuencia de la selva mediana subperennifolia en la zona de influencia de la Reserva de la Biosfera Calakmul, Campeche, México. (Tesis de Maestría). Centro Agronómico Tropical de Investigación y Enseñanza, Turrialba, Costa Rica.

Chao, A., Chazdon, R. L., Colwell, R. K., \& Shen, T. J. (2006). Abundance-based similarity indices and their estimation when there are unseen species in samples. Biometrics, 62, 361-371.

Colwell, R. K. (2008). EstimateS: Statistical estimation of species richness and shared species from samples. Version 8. University of Connecticut. Recuperado de http://www.viceroy.eeb.uconn.edu/estimates.

CONABIO. (2009). Ampliación del Corredor Biológico Mesoamericano-México, en los estados de Tabasco, Oaxaca y Veracruz (Informe Técnico Interno). México D.F., México: Comisión Nacional para el Conocimiento y Uso de la Biodiversidad.

Daily, G. C., Ehrlich, P. R., \& Sánchéz-Azofeifa, G. A. (2001). Countryside biogeography: use of humandominated habitats by the avifauna of Southern Costa Rica. Ecological Applications, 11(1), 1-13.

Díaz-Gallegos, J. R., Mas, J. F., \& Velázquez-Montes, A. (2008). Monitoreo de los patrones de deforestación en el Corredor Biológico Mesoamericano, México. Interciencia, 33(12), 882-890.

Diario Oficial de la Federación. (2010). Norma oficial mexicana NOM-059-SEMARNAT-2010, Protección ambiental-Especies nativas de México de flora y fauna silvestres-Categorías de riesgo y especificaciones para su inclusión, exclusión o cambio- Lista de especies en riesgo. Diario Oficial de la Federación, 30 de Diciembre de 2010. 78 p. Recuperado de www. profepa.gob.mx/innovaportal/file/435/1/NOM_059SEMARNAT_2010.pdf.

Domínguez-Cervantes, E. (2009). Conectividad biológica y social: Zonas de influencia de las áreas naturales protegidas (Serie Conocimientos 5). México D.F., México: Corredor Biológico Mesoamericano México, Comisión Nacional para el Conocimiento y Uso de la Biodiversidad.

Dufrene, M., \& Legendre, P. (1997). Species assemblages and indicator species: The need for a flexible asymmetrical approach. Ecological Monographs, 67(3), 345-366.

Ehrlich, P. R. (1988). The loss of diversity: causes and consequences (pp. 21-27). In E. O. Wilson (Ed.), Biodiversity. Washington DC., USA: National Academy Press.

Estrada, A., Coates-Estrada, R., \& Merrit, D. A. (1997). Anthropogenic landscape changes and avian diversity at Los Tuxtlas, Mexico. Biodiversity and Conservation, 6, 19-43. 
Estrada, A., Cammarano, P., \& Coates-Estrada, R. (2000). Bird species richness in vegetation fences and in strips of residual rain forest vegetation at Los Tuxtlas, Mexico. Biodiversity and Conservation, 9, 1399-1416

Ferguson, B. G., Vandermeer, J., Morales, H., \& Griffith, D. (2003). Post-agricultural succession in El Petén, Guatemala. Conservation Biology, 17(3), 818-828.

Ferguson, B. G. \& Griffith, D. M. (2004). Tecnología agrícola y conservación biológica en El Petén, Guatemala. Manejo Integrado de Plagas y Agroecología, $72,72-85$.

Gillespie, T. W. \& Walter, H. (2001). Distribution of bird species richness at a regional scale in tropical dry forest of Central America. Journal of Biogeography, 28, 651-662.

Gillespie, C. (2002). Latitudinal extent and natural history characteristic in birds of Nicaragua. Global Ecology and Biogeography, 11, 411-417.

Gliessman, S. R., Espinosa, R., \& Amador, M. F. (1981). The ecological basis for the application of traditional agricultural technology in the management of tropical agroecosystems. Agro-Ecosystems, 7, 173-185.

Gomiero, T., Pimentel, D., \& Paoletti, M. G. (2011). Is there a need for a more sustainable agriculture? Critical Reviews in Plant Sciences, 30(1-2), 6-23.

González-Valdivia, N., Ochoa-Gaona, S., Rangel-Ruiz, L. J., Gamboa-Aguilar, J., Pozo, C., Ferguson, B. G., Kampichler, C., Cambranis, E., Lara, O., Pérez-Hernández, I., \& Ponce-Mendoza, A. (2010). Gasterópodos terrestres asociados a un paisaje agropecuario y a un referente ecológico en el Sureste de México (pp. 90-1229). In L. J. Rangel-Ruiz, J. Gamboa-Aguilar, S. L. Arriaga-Weiss, \& W. M. Contreras-Sánchez (comp.), Perspectivas en Malacología Mexicana. Villahermosa, Tabasco, México: Universidad Juárez Autónoma de Tabasco.

González-Valdivia, N., Ochoa-Gaona, S., Pozo, C., Ferguson, B. G., Rangel-Ruiz, L. J., Arriaga-Weiss, S L., Ponce-Mendoza, A., \& Kampichler, C. (2011). Indicadores ecológicos de hábitat y biodiversidad: perspectiva multitaxonómica. Revista de Biología Tropical, 59(3), 1433-1451.

González-Valdivia, N., Ochoa-Gaona, S., Ferguson, B. G., Pozo, C., Kampichler, C., \& Pérez-Hernández, I. (2012a). Análisis comparativo de la estructura, diversidad y composición de comunidades arbóreas de un paisaje agropecuario en Tabasco, México. Revista Mexicana de Biodiversidad, 83, 83-99.

González-Valdivia, N. A., Ochoa-Gaona, S., ArriagaWeiss, S. L., Ferguson, B. G., Kampichler, C., \& Pozo, C. (2012b). Ensambles de aves diurnas a través de un gradiente de perturbación en un paisaje cultural en el Sureste de México. Acta Zoológica Mexicana. Nueva Serie, 28(2), 237-269.
González-Valdivia, N. A., González-Escolástico, G., Barba, E., Hernández-Daumás, S., \& Ochoa-Gaona, S. (2013). Mirmecofauna asociada con sistemas agroforestales en el Corredor Biológico Mesoamericano en Tabasco, México. Revista Mexicana de Biodiversidad, 83, 905-928.

Guevara, S. \& Laborde, J. (1993). Monitoring seed dispersal at isolated standing trees in tropical pastures: consequences for local species availability. Vegetatio, 107/108, 319-338.

Hammer, Ø., Harper, D., \& Ryan, P. (2001). Past: Paleontological Statistics Software Package for education and data analysis. Paleontología Electrónica, 4, 1-9.

Harvey, C. A \& Haber, W. (1999). Remnant trees and the conservation of biodiversity in Costa Rican pastures. Agroforestry Systems, 44, 37-68.

Harvey, C. A., Tucker, N. I. J., \& Estrada, A. (2004). Live fences, isolated trees and windbreaks: tools for conserving biodiversity in fragmented tropical landscapes (pp. 261-289). In G. Schroth, G. A. B. da Fonseca, C. A. Harvey, C. Gascon, H. L.Vasconcelos, \& A. M. N. Izac (Eds.). Agroforestry and biodiversity conservation in tropical landscapes. Chapter 11. Washington DC., USA: Island Press.

Harvey, C. A., Villanueva, C., Villacis, J., Chacón, M., Muñoz, D., López, M., Ibrahim, M., Gómez, R., Taylor, R., Martínez, J., Navas, A., Sáenz, J., Sánchez, D., Medina, A., Vílchez, S., Hernández, B., Pérez, A., Ruiz, F., López, F., Lang, I., \& Sinclair, F. L. (2005). Contribution of live fences to the ecological integrity of agricultural landscapes. Agriculture, Ecosystems and Environment, 111, 200-230.

Harvey, C. A., Medina, A., Merlo-Sánchez, D., Vílchez, S., Hernández, B., Saenz, J. C., Maes, J. M., Casanoves, F., \& Sinclair, F. L. (2006). Patterns of animal diversity in different forms of tree cover in agricultural landscapes. Ecological Applications, 16(5), 1986-1999.

Harvey, C. A. \& González-Villalobos, J. A. (2007). Agroforestry systems conserve species rich but modified assemblages of tropical birds and bats. Biodiversity and Conservation, 16, 2257-2292.

Harvey, C. A., Komar, O., Chazdon, R., Ferguson, B., Finegan, B., Griffith, D., Martínez-Ramos, M., Morales, H., Nigh, R., Soto-Pinto, L., Van Breugel, M., \& Wishnie, M. (2008). Integrating agricultural landscapes with biodiversity conservation in the Mesoamerican hotspot. Conservation Biology, 22(1), 8-15.

Howell, S., \& Webb, S. (1995). A guide to the birds of México and northern Central America. Oxford, New York, USA: Oxford University Press.

Independent Evaluation Group-World Bank. 2011. El Corredor Biológico Mesoamericano. Independent Evaluation Group-World Bank, Banco Mundial 
(Análisis de Programa Regional 5). Washington DC., USA.

Jiménez-Ferrer, G., Pérez-López, H., Soto-Pinto, L., Nahed-Toral, J., Hernández-López, L., \& Carmona, J. (2007). Livestock, nutritive value and local knowledge of fodder trees in fragment landscapes in Chiapas, Mexico. Interciencia, 32(4), 274-280.

Jiménez-Ferrer, G., Velasco-Pérez, R., Uribe-Gómez, M., \& Soto-Pinto, L. (2008). Ganadería y conocimiento local de árboles y arbustos forrajeros de la selva Lacandona, Chiapas, México. Zootecnia Tropical, 26(3), 333-337.

Koller-González, J. M., Arriaga-Weiss, S. L., \& Maldonado-García, N. M. (2010). Aves asociadas a sistemas silvopastoriles en la región de la sierra de Tabasco, México (p. 82). In K. Renton, M. A. Martínez-Morales, R. Pineda-López, J. F. Ornelas, O. Rojas-Soto, \& R. Díaz-Valenzuela (org.). Libro de resúmenes $X$ Congreso para el estudio y la conservación de aves en México (CECAM). Xalapa, Veracruz, México.

Legendre, P. (2007). Studying beta diversity: ecological variation partitioning by multiple regression and canonical analysis. Journal of Plant Ecology, 1(1), 3-8.

Magurran, A. E. (1988). Ecological diversity and its measurement. Princeton, New Jersey, USA: Princeton University Press.

Maldonado, M. N., Grande, D. J., Fuentes, E. E., Hernández, S., Pérez-Gil, F., \& Gómez, A. (2008). Los sistemas silvopastoriles de la región tropical húmeda de México: el caso de Tabasco. Zootecnia Tropical, 26(3), 305-308.

Manjarrez-Muñoz, B., Hernández-Daumás, S., de Jong, B. H. J., Nahed-Toral, J., Vallejo, O. O. D., \& Salvatierra-Zaba, E. B. (2007). Configuración territorial y perspectivas de ordenamiento de la ganadería bovina en los municipios de Balancán y Tenosique, Tabasco. Investigaciones Geográficas, 64, 90-115.

Maridinou, E., Finegan, B., Jiménez-Ferrer, G., Delgado, D., \& Casanoves, F. (2013). Concepts and a methodology for evaluating environmental services from trees of small farms in Chiapas, México. Journal of Environmental Management, 114, 115-124.

Martínez-Encino, C., Villanueva-López, G., \& CasanovaLugo, F. (2013). Densidad y composición de árboles dispersos en potreros en la Sierra de Tabasco, México. Agrociencia, 47(5), 483-496.

Masera, O. R., Ordóñez, M. J., \& Dirzo, R. (1997). Carbon emission from Mexican forest: current situation and long-term scenarios. Climatic Change, 35, 265-295.

McCune, B., \& Mefford, M. J. (1999). Multivariate Analysis of Ecological Data Version 4.25. MjM Software, Gleneden Beach, Oregon, USA.
Moreno, C. E. (2001). Métodos para medir la biodiversidad. M \&T Manuales y Tesis SEA Vol. 1. Zaragoza, España.

Morrison, E. (1999). Introduction to SPSS. Center for Social Science Computation and Research, University of Washington, Washington, USA.

Myers, N. (1988). Tropical forests and their species going, going...? (pp. 28-35). In E .O. Wilson \& F. M. Peter (Eds.), Biodiversity. Washington DC., USA: National Academy Press.

Nair, P. K. R. (1985). Classification of agroforestry systems. Agroforestry Systems, 3, 97-128.

Ochoa-Gaona, S. \& González-Espinosa, M. (2000). Land use and deforestation in the highlands of Chiapas, México. Applied Geography, 20, 17-42.

Ochoa-Gaona, S., Pérez-Hernández, I., \& Jiménez-Pérez, N. C. (2008). Descripción de las especies de árboles más comunes de la sierra de Tenosique, Tabasco, México. El Colegio de la Frontera Sur, FOMIX CONACYT. Tabasco.

Ochoa-Gaona, S., Zamora-Cornelio, L. F., Cabrera-Pérez, S., González-Valdivia, N. A., Pérez-Hernández, I., \& López-Moreno, V. (2012). Flora leñosa útil de la sierra de Tenosique, Tabasco. El Colegio de la Frontera Sur, Villahermosa, Tabasco.

Pérez-Ramírez, I., Van der Wal, H., \& Ishiki-Ishihara, M. (2012). Plantas en recipientes en los huertos familiares de Tabasco. Secretaría de Recursos Naturales y Protección Ambiental y El Colegio de la Frontera Sur, Villahermosa, Tabasco, México.

Peterson, R. T. \& Chalif, E. L. (2006). Aves de México: Guía de campo. Diana: México D.F., México.

Phillips, O. L., Hall, P., Gentry, A. H., Sawyer, S. A., \& Vázquez, R. (1994). Dynamics and species richness of tropical rain forests. Proceedings of the National Academy of Sciences, 91, 2805-2809.

Pinto-Ruiz, L., Hernández, D., Gómez, H., Cobos, M. A., Quiroga, R., \& Pezo, D. (2010). Árboles forrajeros de tres regiones ganaderas de Chiapas: usos y características nutricionales. Universidad y Ciencia, 26(1), 19-31.

PNUMA-CMCM. (2011). Lista de especies CITES (CDROM), Parte 1. Secretaría de la Convención sobre el comercio internacional de especies amenazadas de fauna y flora silvestres, Ginebra, Suiza y Programa de Naciones Unidas para el Medio Ambiente-Centro de Monitoreo de la Conservación Mundial, Cambridge, Reino Unido.

Ramírez-González, A. (2006). Ecología: Métodos de muestreo y análisis de poblaciones y comunidades. Pontificia Universidad Javeriana, Bogotá. Bogotá, Colombia.

Ramírez, G. (2003). El Corredor Biológico Mesoamericano. Biodiversitas, 47, 1-3. 
Rentería-Garita, C. A. (2009). La colonización, adaptación y desarrollo económico de la selva a través de la ganadería: el caso de la frontera Tabasco-Chiapas, México. Iberoforum, 7, 66-93.

Robinson,W. D. (2001). Changes in abundance of birds in a Neotropical forest fragment over 25 years: a review. Animal Biodiversity and Conservation, 24(2), 51-65.

Ruiz-Rodríguez, J. M. (2000). Los sistemas silvopastoriles: Opción sustentable de los recursos naturales tropicales. Universidad Autónoma de Chapingo, Centro Regional Universitario del Sureste (CRUSE), Informe Final SNIB-CONABIO Proyecto No. M-095, México, D.F., México.

Sans, F. X. (2007). La diversidad de los agroecosistemas. Ecosistemas, 16(1), 44-49.

Secretaría de Desarrollo Social y Protección al Ambiente. (2006). Programa de ordenamiento ecológico del estado de Tabasco. Secretaría de Desarrollo Social y Protección al Ambiente, Gobierno de Tabasco, Villahermosa, Tabasco, México.

Shankar-Raman, T., Rawat, G., \& Johnsingh, A. (1998). Recovery of tropical rainforest avifauna in relation to vegetation succession following shifting cultivation in Mizoram, north-east India. Journal of Applied Ecology, 35, 214-231.
Sinclair, F. L. (1999). A general classification of agroforestry practice. Agroforestry Systems, 46, 161-180.

Spellerberg, I. F. (2005). Monitoring Ecological Change. England: Cambridge University.

Toledo, V. M., Batis, A. I., Becerra, R., Martínez, E., \& Ramos, C. (1995). La selva útil: Etnobotánica cuantitativa de los grupos indígenas del trópico húmedo de México. Interciencia, 20(4), 177-187.

Van der Wal, H., Peña-Álvarez, B., Arriaga-Weiss, S. L., \& Hernández-Daumás, S. (2012). Species, functional groups, and habitat preferences of birds in five agroforestry classes in Tabasco, Mexico. Wilson Journal of Ornithology, 124, 558-571.

Van Perlo, B. (2006). Birds of Mexico and Central America. Illustrated Checklist. New Jersey, USA: Princeton University Press.

Winker, K, Arriaga-Weiss, S. L., Trejo, J. L., \& Escalante, P. (1999). Notes on the avifauna of Tabasco. The Wilson Bulletin, 111, 229-235.

Zuur, A., Ieno, E., \& Smith, G. (2007). Analyzing ecological data (pp. 163-179). In M. Gail, K. Krickeberg, J. Samet, A. Tsiatis, \& W. Wong (Eds.), Statistics for biology and health. New York, USA: Springer. 


\section{APÉNDICE}

Registros de las especies de aves por sistema silvopastoril, sitio y correlaciones significativas entre las especies y las variables ambientales

\section{APPENDIX}

Records of birds' species for silvopastoral system, site, and significant correlations between bird species and environmental variables

\begin{tabular}{|c|c|c|c|c|c|c|c|c|c|c|c|c|c|c|c|c|}
\hline \multirow{3}{*}{ Gremio } & \multirow{3}{*}{ Sistema silvopastoril } & \multicolumn{6}{|c|}{ Municipio } & \multicolumn{9}{|c|}{ Correlaciones de Pearson $(\mathrm{p}<0.05)$} \\
\hline & & \multicolumn{2}{|c|}{ Huimanguillo } & \multicolumn{2}{|c|}{ Tacotalpa } & \multicolumn{2}{|c|}{ Tenosique } & \multicolumn{4}{|c|}{ Variables estructurales } & \multicolumn{5}{|c|}{ Variables ambientales } \\
\hline & & $\mathrm{AL}$ & $\mathrm{ADP}$ & $\mathrm{AL}$ & ADP & $\mathrm{AL}$ & ADP & DA & $\mathrm{AB}$ & $\mathrm{C}$ & $\mathrm{AT}$ & Alt & HRS & HRL & TS & $\mathrm{TL}$ \\
\hline & Familia/Especie & & & & & & & & & & & & & & & \\
\hline & Familia Phalacrocoracidae & & & & & & & & & & & & & & & \\
\hline \multirow[t]{2}{*}{$\mathrm{CA}$} & Phalacrocorax olivaceus & 4 & 0 & 0 & 0 & 0 & 0 & & & & & & & & & \\
\hline & Familia Ardeidae & & & & & & & & & & & & & & & \\
\hline CA & Ardea herodias & 0 & 1 & 0 & 0 & 0 & 0 & & & & & & & & & \\
\hline $\mathrm{CA}$ & Botaurus lentiginosus ${ }^{\mathrm{A}}$ & 4 & 1 & 0 & 0 & 0 & 0 & & & & & & & & & \\
\hline Gan & Bubulcus ibis & 5 & 40 & 9 & 2 & 5 & 9 & & & & & & & & & \\
\hline $\mathrm{CA}$ & Butorides striatus & 0 & 1 & 0 & 0 & 0 & 0 & & & & & & & & & \\
\hline $\mathrm{CA}$ & Butorides virescens & 2 & 1 & 0 & 0 & 0 & 0 & & & & & -0.17 & 0.18 & & & \\
\hline $\mathrm{CA}$ & Casmerodius albus & 4 & 6 & 0 & 1 & 0 & 0 & & & & & -0.22 & & & & \\
\hline $\mathrm{CA}$ & Cochlearius conchlearius & 1 & 0 & 0 & 0 & 0 & 0 & & & & & & & & & \\
\hline $\mathrm{CA}$ & Egretta caerulea & 2 & 0 & 0 & 0 & 0 & 0 & & & & & & & & & \\
\hline $\mathrm{CA}$ & Egretta thula & 15 & 23 & 0 & 0 & 0 & 0 & & & & & -0.24 & & & & \\
\hline \multirow[t]{2}{*}{$\mathrm{CA}$} & Nyctycorax nyctycorax & 1 & 0 & 0 & 0 & 0 & 0 & & & & & & & & & \\
\hline & Familia Cathartidae & & & & & & & & & & & & & & & \\
\hline \multirow[t]{2}{*}{ Car } & Coragyps atratus & 1 & 15 & 4 & 1 & 0 & 0 & & & & & & & & & \\
\hline & Familia Anatidae & & & & & & & & & & & & & & & \\
\hline $\mathrm{CA}$ & Cairina moschata ${ }^{\mathrm{PE}, \mathrm{C}-\mathrm{III}}$ & 0 & 4 & 0 & 0 & 0 & 0 & & & & & & & & & \\
\hline \multirow[t]{2}{*}{$\mathrm{CA}$} & Dendrocygna autumnalis *C-III & 3 & 19 & 0 & 0 & 0 & 0 & & & 0.17 & & -0.27 & & & & \\
\hline & Familia Accipitridae & & & & & & & & & & & & & & & \\
\hline $\mathrm{RD}$ & Chondrohierax uncinatus ${ }^{\mathrm{P}, \mathrm{C}-\mathrm{I}}$ & 2 & 13 & 2 & 4 & 4 & 2 & & & & & & & & & \\
\hline $\mathrm{RD}$ & Ictinia plumbea $^{\mathrm{Pr}, \mathrm{C}-\mathrm{II}}$ & 0 & 1 & 0 & 0 & 0 & 1 & & & & & & & & & \\
\hline $\mathrm{RD}$ & Busarellus nigricollis $^{\mathrm{Pr}, \mathrm{C}-\mathrm{II}}$ & 1 & 0 & 0 & 0 & 0 & 0 & & & & & & & & & \\
\hline \multirow[t]{2}{*}{$\mathrm{RD}$} & Buteo nitidus $^{\mathrm{C}-\mathrm{II}}$ & 0 & 0 & 0 & 1 & 0 & 1 & -0.17 & -0.18 & & & & & & & \\
\hline & Familia Falconidae & & & & & & & & & & & & & & & \\
\hline $\mathrm{RD}$ & Falco columbarius ${ }^{\mathrm{C}-\mathrm{II}}$ & 2 & 0 & 0 & 0 & 0 & 0 & & & & & & & & & \\
\hline $\mathrm{RD}$ & Falco femoralis ${ }^{\mathrm{Pr}, \mathrm{C}-\mathrm{II}}$ & 0 & 2 & 0 & 0 & 0 & 0 & & & & & & & & & \\
\hline \multirow[t]{2}{*}{$\mathrm{RD}$} & Herpethoteres cachinnans ${ }^{\mathrm{C}-\mathrm{II}}$ & 2 & 2 & 0 & 1 & 0 & 1 & & & & & & & & & \\
\hline & Familia Cracidae & & & & & & & & & & & & & & & \\
\hline \multirow[t]{2}{*}{ Gfa } & Ortalis vetula ${ }^{\mathrm{C}-\mathrm{III}}$ & 13 & 6 & 6 & 6 & 2 & 0 & & & & & & & & & \\
\hline & Familia Rallidae & & & & & & & & & & & & & & & \\
\hline $\mathrm{CA}$ & Aramides cajaneus & 0 & 2 & 0 & 0 & 0 & 0 & & & & & & & & & \\
\hline \multirow[t]{2}{*}{$\mathrm{CA}$} & Pophyrio martinicus & 1 & 0 & 0 & 0 & 0 & 0 & & & & & & & & & \\
\hline & Familia Aramidae & & & & & & & & & & & & & & & \\
\hline \multirow[t]{2}{*}{$\mathrm{CA}$} & Aramus guarauna $^{\mathrm{A}}$ & 0 & 1 & 0 & 0 & 0 & 0 & & & & & & & & & \\
\hline & Familia Jacanidae & & & & & & & & & & & & & & & \\
\hline \multirow[t]{2}{*}{ CA } & Jacana spinosa & 3 & 1 & 0 & 0 & 0 & 0 & & & & & & & & & \\
\hline & Familia Columbidae & & & & & & & & & & & & & & & \\
\hline Gft & Columbina inca & 4 & 4 & 0 & 0 & 0 & 0 & & & & & -0.19 & & & & \\
\hline
\end{tabular}


APÉNDICE (Continuación) / APPENDIX (Continued)

\begin{tabular}{|c|c|c|c|c|c|c|c|c|c|c|c|c|c|c|c|}
\hline \multirow{3}{*}{ Gremio } & \multirow{3}{*}{ Sistema silvopastoril } & \multicolumn{6}{|c|}{ Municipio } & \multicolumn{8}{|c|}{ Correlaciones de Pearson $(\mathrm{p}<0.05)$} \\
\hline & & \multicolumn{2}{|c|}{ Huimanguillo } & \multicolumn{2}{|c|}{ Tacotalpa } & \multicolumn{2}{|c|}{ Tenosique } & \multicolumn{4}{|c|}{ Variables estructurales } & \multicolumn{4}{|c|}{ Variables ambientales } \\
\hline & & $\mathrm{AL}$ & ADP & $\mathrm{AL}$ & $\mathrm{ADP}$ & $\mathrm{AL}$ & ADP & DA & $\mathrm{AB}$ & $\mathrm{C}$ & AT & Alt & HRS & HRL TS & $\mathrm{TL}$ \\
\hline Gft & Columbina minuta & 8 & 7 & 0 & 1 & 2 & 4 & & & & & & & & -0.17 \\
\hline Gft & Columbina talpacoti & 0 & 0 & 0 & 0 & 0 & 1 & & & & & & & & \\
\hline Gft & Leptotila verreauxi & 0 & 0 & 1 & 1 & 0 & 0 & & & & & & & & -0.18 \\
\hline Gft & Patagioenas cayennesis & 0 & 0 & 0 & 0 & 1 & 0 & & & & & & & & \\
\hline Gft & Patagioenas flavirostris* & 11 & 19 & 1 & 2 & 4 & 2 & & & & & -0.27 & & & \\
\hline Gft & Patagioenas nigrirostris ${ }^{* \mathrm{Pr}}$ & 0 & 0 & 0 & 1 & 0 & 0 & & & & & & & & \\
\hline \multirow[t]{2}{*}{ Gft } & Zenaida asiatica & 1 & 0 & 1 & 0 & 0 & 2 & & & & & & & & \\
\hline & Familia Psittacidae & & & & & & & & & & & & & & \\
\hline Gfa & Amazona albifrons $^{\mathrm{Pr}, \mathrm{C}-\mathrm{II}}$ & 0 & 0 & 2 & 12 & 2 & 0 & & -0.18 & & & & & -0.25 & \\
\hline Gfa & Amazona autumnalis ${ }^{\mathrm{C}-\mathrm{II}}$ & 2 & 6 & 10 & 6 & 0 & 0 & & & & & & & -0.17 & \\
\hline Gfa & Amazona farinosa ${ }^{\mathrm{PE}, \mathrm{C}-\mathrm{II}}$ & 0 & 0 & 2 & 0 & 0 & 0 & & & & & & & & \\
\hline Gfa & Amazona oratrix ${ }^{\mathrm{PE}, \mathrm{C}-\mathrm{I}}$ & 2 & 5 & 0 & 0 & 0 & 0 & & & & & -0.21 & & & \\
\hline Gfa & Aratinga holochlora ${ }^{\mathrm{A}, \mathrm{C}-\mathrm{II}}$ & 24 & 2 & 36 & 33 & 2 & 34 & & -0.2 & & & 0.18 & & -0.17 & \\
\hline \multirow[t]{2}{*}{ Gfa } & Pionus senilis ${ }^{\mathrm{A}, \mathrm{C}-\mathrm{II}}$ & 0 & 0 & 0 & 0 & 0 & 4 & & & & & & & & \\
\hline & Familia Cuculidae & & & & & & & & & & & & & & \\
\hline Gan & Crotophaga sulcirostris & 6 & 7 & 6 & 2 & 2 & 11 & & & & & & & & -0.18 \\
\hline \multirow[t]{2}{*}{$\mathrm{OM}$} & Piaya cayana & 0 & 0 & 1 & 0 & 0 & 0 & & & & & & & & \\
\hline & Familia Strigidae & & & & & & & & & & & & & & \\
\hline $\mathrm{RN}$ & Pseudoscops clamator ${ }^{\mathrm{A}, \mathrm{C}-\mathrm{II}}$ & 1 & 0 & 0 & 0 & 0 & 0 & & & & & & & & \\
\hline \multirow[t]{2}{*}{$\mathrm{RD}$} & Glaucidium brasilianum $^{\mathrm{C}-\mathrm{II}}$ & 0 & 1 & 0 & 0 & 0 & 0 & & & & & & & & \\
\hline & Familia Trochilidae & & & & & & & & & & & & & & \\
\hline $\mathrm{N}$ & Amazilia cyanocephala ${ }^{\mathrm{C}-\mathrm{II}}$ & 0 & 0 & 0 & 1 & 0 & 0 & & & & & & & & \\
\hline $\mathrm{N}$ & Amazilia tzacatl ${ }^{\mathrm{C}-\mathrm{II}}$ & 1 & 3 & 3 & 2 & 2 & 0 & & & & & & & & \\
\hline $\mathrm{N}$ & Anthracothorax prevostii ${ }^{\mathrm{C}-\mathrm{II}}$ & 0 & 2 & 0 & 0 & 1 & 2 & & & & & & & & \\
\hline $\mathrm{N}$ & Eupherusa eximia ${ }^{\mathrm{C}-\mathrm{II}}$ & 0 & 0 & 2 & 1 & 0 & 1 & & & & & & & & \\
\hline $\mathrm{N}$ & Hylocharis leucotis & 0 & 0 & 2 & 2 & 1 & 0 & & & & & 0.18 & & & \\
\hline \multirow[t]{2}{*}{$\mathrm{N}$} & Threnetes ruckeri ${ }^{\mathrm{C}-\mathrm{II}}$ & 1 & 0 & 0 & 0 & 0 & 1 & & & & & & & & \\
\hline & Familia Trogonidae & & & & & & & & & & & & & & \\
\hline \multirow[t]{2}{*}{$\mathrm{OA}$} & Trogon melanocephalus & 1 & 2 & 2 & 4 & 1 & 10 & & -0.18 & & & & & & \\
\hline & Familia Momotidae & & & & & & & & & & & & & & \\
\hline \multirow[t]{2}{*}{$\operatorname{Tg}$} & Momotus momota & 0 & 0 & 1 & 0 & 0 & 1 & & & & & & & & \\
\hline & Familia Alcedinidae & & & & & & & & & & & & & & \\
\hline CA & Chloroceryle aenea & 1 & 1 & 0 & 0 & 0 & 0 & & & & & -0.18 & & & \\
\hline CA & Megaceryle alcyon & 0 & 0 & 0 & 0 & 0 & 2 & & & & & & & & \\
\hline \multirow[t]{2}{*}{ CA } & Megaceryle torquata & 4 & 0 & 0 & 0 & 0 & 0 & & & & & -0.18 & & & \\
\hline & Familia Ramphastidae & & & & & & & & & & & & & & \\
\hline Gfa & Pteroglossus torquatus ${ }^{\mathrm{Pr}}$ & 0 & 0 & 0 & 4 & 0 & 0 & & & & & & & & \\
\hline Gfa & Ramphastos sulfuratus ${ }^{\mathrm{A}, \mathrm{C}-\mathrm{II}}$ & 0 & 0 & 2 & 2 & 0 & 0 & & & & & 0.21 & & & \\
\hline & Familia Picidae & & & & & & & & & & & & & & \\
\hline Cro & Dryocopus lineatus & 0 & 2 & 0 & 0 & 2 & 0 & & & & & & & & \\
\hline Cro & Melanerpes aurifrons & 11 & 12 & 4 & 6 & 3 & 14 & & & & & & & & \\
\hline & Familia Furnariidae & & & & & & & & & & & & & & \\
\hline S & Automolus ochrolaemus ${ }^{\mathrm{Pr}}$ & 0 & 0 & 1 & 0 & 0 & 0 & & & & & & & & \\
\hline & Familia Thamnophilidae & & & & & & & & & & & & & & \\
\hline $\mathrm{Pbh}$ & Thamnophilus doliatus & 0 & 1 & 0 & 0 & 0 & 0 & & & & & & & & \\
\hline
\end{tabular}


APÉNDICE (Continuación) / APPENDIX (Continued)

\begin{tabular}{|c|c|c|c|c|c|c|c|c|c|c|c|c|c|c|c|c|}
\hline \multirow{3}{*}{ Gremio } & \multirow{3}{*}{ Sistema silvopastoril } & \multicolumn{6}{|c|}{ Municipio } & \multicolumn{9}{|c|}{ Correlaciones de Pearson $(\mathrm{p}<0.05)$} \\
\hline & & \multicolumn{2}{|c|}{ Huimanguillo } & \multicolumn{2}{|c|}{ Tacotalpa } & \multicolumn{2}{|c|}{ Tenosique } & \multicolumn{4}{|c|}{ Variables estructurales } & \multicolumn{5}{|c|}{ Variables ambientales } \\
\hline & & $\mathrm{AL}$ & $\mathrm{ADP}$ & $\mathrm{AL}$ & $\mathrm{ADP}$ & $\mathrm{AL}$ & ADP & DA & $\mathrm{AB}$ & $\mathrm{C}$ & $\mathrm{AT}$ & Alt & HRS & HRL & TS & $\mathrm{TL}$ \\
\hline & Familia Tyrannidae & & & & & & & & & & & & & & & \\
\hline $\mathrm{Tp}$ & Contopus borealis & 0 & 2 & 0 & 0 & 0 & 0 & & & & & & & & & \\
\hline $\mathrm{Tp}$ & Contopus cinereus & 0 & 0 & 0 & 0 & 1 & 0 & & & & & & & & & \\
\hline $\mathrm{Tp}$ & Contopus pertinax & 0 & 0 & 0 & 0 & 1 & 0 & & & & & & & & & \\
\hline $\mathrm{Tp}$ & Contopus sordidulus* & 1 & 0 & 0 & 2 & 0 & 3 & & -0.18 & & & & & & & \\
\hline $\mathrm{Tp}$ & Contopus virens & 2 & 0 & 1 & 0 & 0 & 0 & & & & & & & & & \\
\hline $\mathrm{Tp}$ & Elaenia flavogaster & 1 & 0 & 0 & 1 & 0 & 2 & & & & & & & & 0.23 & \\
\hline $\mathrm{Tp}$ & Empidonax affinis & 0 & 0 & 0 & 1 & 0 & 0 & & & & & & & & & \\
\hline $\mathrm{Tp}$ & Empidonax albigularis & 0 & 0 & 1 & 0 & 0 & 0 & & & & & & & & & \\
\hline $\mathrm{Tp}$ & Empidonax alnorum & 0 & 0 & 1 & 0 & 0 & 0 & & & & & & & & & \\
\hline $\mathrm{Tp}$ & Empidonax flavescens & 0 & 0 & 1 & 0 & 1 & 1 & & & & & & & & & \\
\hline $\mathrm{Tp}$ & Empidonax traillii & 0 & 1 & 0 & 1 & 0 & 1 & & & & 0.2 & & -0.17 & & & \\
\hline $\mathrm{Tp}$ & Empidonax virescens & 2 & 0 & 1 & 0 & 0 & 1 & & & & & & & & & \\
\hline $\mathrm{Tg}$ & Myiarchus crinitus & 2 & 0 & 0 & 2 & 1 & 3 & & & & & & & & & \\
\hline $\mathrm{Tg}$ & Myiarchus nuttingi & 0 & 0 & 1 & 0 & 0 & 0 & & & & & & & & & \\
\hline $\mathrm{Tg}$ & Myiarchus tuberculifer & 0 & 0 & 1 & 0 & 0 & 1 & & & & & & & & & \\
\hline $\mathrm{Tg}$ & Myiodynastes luteiventris & 0 & 0 & 1 & 1 & 0 & 3 & & & & & 0.17 & & & & \\
\hline $\mathrm{Tg}$ & Myiodynastes maculatus & 0 & 0 & 0 & 0 & 2 & 0 & & & & & & & & & \\
\hline $\mathrm{Tg}$ & Myiozetetes similis* & 2 & 4 & 4 & 3 & 24 & 30 & & & & 0.18 & & -0.22 & 0.28 & & -0.25 \\
\hline $\mathrm{Tg}$ & Pitangus sulphuratus & 12 & 8 & 2 & 7 & 3 & 3 & & & & & & & & & \\
\hline $\mathrm{Tp}$ & Pyrocephalus rubinus & 2 & 0 & 0 & 0 & 0 & 2 & & & & & & & & & \\
\hline $\mathrm{Pbh}$ & Rynchocyclus brevirostris & 0 & 0 & 1 & 0 & 2 & 0 & & & & & & & & & \\
\hline $\mathrm{Pbh}$ & Todirostrum cinereum & 0 & 0 & 0 & 0 & 2 & 4 & & & & & & & 0.2 & & \\
\hline $\mathrm{Tg}$ & Tyrannus forficatus & 0 & 0 & 0 & 0 & 0 & 2 & & & & & & & & & \\
\hline $\mathrm{Tg}$ & Tyrannus melancholicus* & 33 & 27 & 0 & 0 & 11 & 5 & & 0.32 & & & -0.46 & & 0.27 & & \\
\hline $\mathrm{Tg}$ & Tyrannus savana & 2 & 1 & 0 & 0 & 0 & 0 & & & & & & & & & \\
\hline $\mathrm{Tg}$ & Tyrannus tyrannus & 2 & 0 & 0 & 0 & 0 & 0 & & & & & & & & & \\
\hline \multirow[t]{2}{*}{$\mathrm{Tg}$} & Tyrannus verticalis & 2 & 1 & 0 & 0 & 2 & 0 & & & & & & & & & \\
\hline & Familia Tytiridae & & & & & & & & & & & & & & & \\
\hline Pam & Pachyramphus agliae & 0 & 0 & 1 & 0 & 0 & 0 & & & & & & & & & \\
\hline \multirow[t]{2}{*}{ Gfa } & Tityra semifasciata & 0 & 0 & 1 & 5 & 2 & 4 & & & & & & -0.22 & & & \\
\hline & Familia Vireonidae & & & & & & & & & & & & & & & \\
\hline Pam & Vireo bellii & 0 & 0 & 0 & 0 & 0 & 2 & & & & & & & & & \\
\hline Pam & Vireo flavoviridis & 0 & 1 & 2 & 0 & 2 & 0 & & & & & & & & & \\
\hline Pam & Vireo gilvus & 0 & 2 & 0 & 0 & 0 & 0 & & & & & & & & & \\
\hline Pam & Vireo griseus & 0 & 0 & 0 & 1 & 0 & 1 & & & & & & & & & \\
\hline Pam & Vireo huttoni & 0 & 0 & 0 & 0 & 0 & 1 & & & & & & & & & \\
\hline Pam & Vireo hypochryseus & 0 & 2 & 0 & 0 & 1 & 0 & & & & 0.21 & & -0.19 & & & \\
\hline Pam & Vireo leucophrys & 0 & 0 & 0 & 0 & 1 & 4 & & & -0.17 & & & & & & \\
\hline \multirow[t]{2}{*}{ Pam } & Vireo philadelphicus & 0 & 0 & 0 & 0 & 0 & 4 & & & & & & -0.17 & & & \\
\hline & Familia Corvidae & & & & & & & & & & & & & & & \\
\hline \multirow[t]{2}{*}{$\mathrm{OM}$} & Psilorhinus morio & 34 & 38 & 33 & 33 & 20 & 13 & & & & & & & & & \\
\hline & Familia Troglodytidae & & & & & & & & & & & & & & & \\
\hline Gfa & Campylorhynchus zonatus & 6 & 8 & 1 & 0 & 0 & 0 & & & & -0.17 & & 0.17 & & -0.18 & \\
\hline $\mathrm{S}$ & Pheugopedius maculipectus & 0 & 1 & 0 & 0 & 0 & 0 & & & & & & & & & \\
\hline
\end{tabular}


APÉNDICE (Continuación) / APPENDIX (Continued)

\begin{tabular}{|c|c|c|c|c|c|c|c|c|c|c|c|c|c|c|c|c|}
\hline \multirow{3}{*}{ Gremio } & \multirow{3}{*}{ Sistema silvopastoril } & \multicolumn{6}{|c|}{ Municipio } & \multicolumn{9}{|c|}{ Correlaciones de Pearson $(\mathrm{p}<0.05)$} \\
\hline & & \multicolumn{2}{|c|}{ Huimanguillo } & \multicolumn{2}{|c|}{ Tacotalpa } & \multicolumn{2}{|c|}{ Tenosique } & \multicolumn{4}{|c|}{ Variables estructurales } & \multicolumn{5}{|c|}{ Variables ambientales } \\
\hline & & $\mathrm{AL}$ & ADP & $\mathrm{AL}$ & $\mathrm{ADP}$ & $\mathrm{AL}$ & ADP & DA & $\mathrm{AB}$ & $\mathrm{C}$ & AT & Alt & HRS & HRL & TS & $\mathrm{TL}$ \\
\hline $\mathrm{S}$ & Pheugopedius modestus & 0 & 0 & 1 & 2 & 0 & 0 & & & & & & & & & \\
\hline S & Troglodytes aedon & 0 & 0 & 1 & 1 & 0 & 0 & & & & & 0.17 & & & & \\
\hline \multirow[t]{2}{*}{ S } & Troglodytes musculus & 0 & 2 & 4 & 0 & 3 & 1 & & & & & & & & & \\
\hline & Familia Polioptilidae & & & & & & & & & & & & & & & \\
\hline Gft & Polioptila caerulea & 0 & 0 & 0 & 0 & 0 & 2 & & & & & & & & & \\
\hline Pam & Polioptila plumbea $a^{\mathrm{Pr}}$ & 0 & 1 & 0 & 0 & 2 & 0 & & & & & & & & & \\
\hline \multirow[t]{2}{*}{$\mathrm{Pbh}$} & Ramphocaenus melanurus & 0 & 0 & 3 & 0 & 0 & 0 & & & & & & & & & \\
\hline & Familia Turdidae & & & & & & & & & & & & & & & \\
\hline $\mathrm{Pbh}$ & Catharus mexicanus $^{\mathrm{Pr}}$ & 0 & 0 & 0 & 2 & 0 & 0 & & & & 0.19 & 0.17 & & & & \\
\hline $\mathrm{Pbh}$ & Catharus ustulatus & 0 & 0 & 0 & 0 & 1 & 0 & & & & & & & & & \\
\hline Gft & Myadestes unicolor ${ }^{\mathrm{A}}$ & 0 & 0 & 0 & 1 & 0 & 0 & & & & & & & & & \\
\hline \multirow[t]{2}{*}{ Gft } & Turdus grayi & 8 & 3 & 2 & 0 & 2 & 4 & & & & & & & & & \\
\hline & Familia Mimidae & & & & & & & & & & & & & & & \\
\hline Gfa & Dumetella caroliniensis & 0 & 0 & 0 & 1 & 0 & 0 & & & & & & & & & \\
\hline \multirow[t]{2}{*}{ Gft } & Mimus gilvus & 0 & 0 & 0 & 0 & 0 & 2 & & & & & & & & & \\
\hline & Familia Parulidae & & & & & & & & & & & & & & & \\
\hline $\mathrm{Pbh}$ & Basileuterus culicivorus & 0 & 0 & 0 & 1 & 0 & 1 & & & & 0.17 & & & & & \\
\hline $\mathrm{Pbh}$ & Geothlypis philadelphia & 0 & 1 & 0 & 0 & 0 & 0 & & & & & & & & & \\
\hline $\mathrm{Pbh}$ & Geothlypis poliocephala & 0 & 1 & 0 & 0 & 0 & 4 & & & & & & & & & \\
\hline $\mathrm{Pbh}$ & Geothlypis trichas & 0 & 0 & 0 & 0 & 2 & 1 & & & & & & & & & \\
\hline $\mathrm{S}$ & Helmitheros vermivorus & 0 & 4 & 4 & 0 & 3 & 0 & & & & & & & & & \\
\hline Pam & Mniotilta varia & 0 & 0 & 1 & 2 & 0 & 2 & & & & & & & & & \\
\hline Pam & Oreothlypis peregrina & 5 & 2 & 2 & 1 & 6 & 2 & & & -0.18 & & & -0.17 & & & \\
\hline Pam & Oreothlypis ruficapilla & 1 & 0 & 0 & 0 & 0 & 0 & & & & & & & & & \\
\hline S & Parkesia motacilla & 0 & 0 & 0 & 1 & 0 & 0 & & & & & & & & & \\
\hline $\mathrm{S}$ & Seiurus auricapilla & 0 & 1 & 0 & 0 & 2 & 0 & & & & & & & & & \\
\hline Pam & Setophaga cerulea & 0 & 0 & 0 & 0 & 0 & 2 & & & & & & -0.17 & & & \\
\hline Pam & Setophaga citrina & 0 & 4 & 0 & 0 & 0 & 0 & & & & & & & & & \\
\hline Pam & Setophaga fusca* & 0 & 0 & 2 & 1 & 0 & 0 & & & & & 0.17 & & -0.18 & 0.18 & \\
\hline Pam & Setophaga magnolia & 0 & 2 & 7 & 4 & 2 & 1 & & & & & 0.21 & & -0.19 & & \\
\hline Pam & Setophaga petechia & 5 & 5 & 3 & 1 & 8 & 3 & & & & & & & & & \\
\hline Pam & Setophaga ruticilla* & 0 & 4 & 7 & 7 & 12 & 13 & & & & & & -0.21 & & & \\
\hline Pam & Setophaga striata & 0 & 0 & 1 & 6 & 0 & 2 & & -0.19 & & & 0.19 & & -0.18 & & \\
\hline Pam & Setophaga towsendi & 0 & 0 & 2 & 5 & 2 & 1 & & & & & & & & & \\
\hline & Incertae sedis (1) & & & & & & & & & & & & & & & \\
\hline $\mathrm{Tp}$ & Coereba flaveola & 0 & 2 & 0 & 0 & 0 & 0 & & & & & & & & & \\
\hline & Incertae sedis (2) & & & & & & & & & & & & & & & \\
\hline Gfa & Saltator atriceps* & 0 & 0 & 5 & 7 & 17 & 12 & & & & & & & & & \\
\hline & Familia Thraupidae & & & & & & & & & & & & & & & \\
\hline $\mathrm{N}$ & Cyanerpes cyaneus & 0 & 0 & 0 & 0 & 0 & 1 & & & & & & & & & \\
\hline Pam & Euphonia gouldi $i^{\mathrm{Pr}}$ & 4 & 0 & 0 & 0 & 0 & 0 & & & & & & & & & \\
\hline Pam & Euphonia hirundinacea & 0 & 2 & 0 & 5 & 3 & 0 & & & & & & & & & \\
\hline $\mathrm{Pbh}$ & Ramphocelus passerinii & 0 & 0 & 1 & 1 & 0 & 0 & & & & & & & & & \\
\hline Gfa & Thraupis abbas & 2 & 0 & 0 & 0 & 1 & 0 & & 0.18 & & & & & & & \\
\hline Gfa & Thraupis episcopus & 2 & 1 & 1 & 2 & 0 & 2 & & & & & & & & & \\
\hline
\end{tabular}


APÉNDICE (Continuación) / APPENDIX (Continued)

\begin{tabular}{|c|c|c|c|c|c|c|c|c|c|c|c|c|c|c|c|c|}
\hline \multirow{3}{*}{ Gremio } & \multirow{3}{*}{ Sistema silvopastoril } & \multicolumn{6}{|c|}{ Municipio } & \multicolumn{9}{|c|}{ Correlaciones de Pearson $(\mathrm{p}<0.05)$} \\
\hline & & \multicolumn{2}{|c|}{ Huimanguillo } & \multicolumn{2}{|c|}{ Tacotalpa } & \multicolumn{2}{|c|}{ Tenosique } & \multicolumn{4}{|c|}{ Variables estructurales } & \multicolumn{5}{|c|}{ Variables ambientales } \\
\hline & & $\mathrm{AL}$ & $\mathrm{ADP}$ & $\mathrm{AL}$ & ADP & $\mathrm{AL}$ & $\mathrm{ADP}$ & DA & $\mathrm{AB}$ & $\mathrm{C}$ & AT & Alt & HRS & HRL & TS & TL \\
\hline & Familia Emberizidae & & & & & & & & & & & & & & & \\
\hline $\mathrm{Pbh}$ & Arremonops chloronotus & 0 & 0 & 0 & 0 & 0 & 3 & & & & & & & & & \\
\hline Gft & Sporophila americana & 6 & 57 & 9 & 19 & 11 & 51 & & & & & & & & & \\
\hline \multirow[t]{2}{*}{ Gft } & Sporophila torqueola & 12 & 41 & 3 & 9 & 6 & 20 & & & & & & & & & \\
\hline & Familia Cardinalidae & & & & & & & & & & & & & & & \\
\hline Gfa & Guiraca caerulea & 0 & 1 & 0 & 0 & 0 & 0 & & & & & & & & & \\
\hline Pam & Granatellus sallaei & 0 & 0 & 1 & 0 & 0 & 0 & & & & & & & & & \\
\hline Gfa & Passerina cyanea & 0 & 0 & 0 & 3 & 0 & 0 & & & & & & & & & \\
\hline Pam & Pheutictus ludovicianus & 1 & 0 & 3 & 2 & 0 & 0 & & & & & & & & & \\
\hline $\mathrm{Pbh}$ & Piranga roseogularis & 0 & 0 & 0 & 2 & 0 & 2 & & & & & & & & & \\
\hline \multirow[t]{2}{*}{$\mathrm{Pbh}$} & Piranga rubra* & 0 & 0 & 3 & 1 & 1 & 0 & & & & & & & & & \\
\hline & Familia Icteridae & & & & & & & & & & & & & & & \\
\hline Gfa & Icterus gularis & 3 & 7 & 3 & 0 & 0 & 0 & & & & -0.17 & & & & & \\
\hline Gfa & Icterus spurius $^{\mathrm{Pr}}$ & 0 & 4 & 13 & 21 & 9 & 25 & & & & & 0.24 & & -0.18 & 0.21 & -0.28 \\
\hline Gfa & Psarocolius montezuma ${ }^{\mathrm{Pr}}$ & 5 & 27 & 3 & 6 & 2 & 3 & & & & & & & & & \\
\hline $\mathrm{OM}$ & Quiscalus mexicanus & 32 & 15 & 4 & 75 & 4 & 1 & & & & & -0.19 & & & & 0.19 \\
\hline Gft & Sturnella magna* & 3 & 9 & 0 & 0 & 0 & 0 & & & & & -0.26 & & & & \\
\hline
\end{tabular}

Estatus de protección según NOM-059-Semarnat (Diario Oficial de la Federación 2010): En superíndice A: amenazada, PE: en peligro de extinción, Pr: protección especial. *Especie indicadora de región fisiográfica en Tabasco (IndVal $>0.70$, p $<0.05$ ). (1) Antes Coerebidae, (2) Antes Cardinalidae. Superíndices C-I, C-II y C-III representan especies enlistadas en los Anexos CITES (PNUMA-CMCM 2011)

Sistema silvopastoril: AL: árboles en linderos, ADP: árboles dispersos en potreros.

G: Gremio de forrajeo: CA: cazador acuático, Car: carroñero, Cro: cazador que busca presas perforando la madera, Gan: cazador asociado a ganado vacuno, Gfa: granívoro-frugívoro arbóreo, Gft: granívoro-frugívoro terrestre, N: nectarívoro, OA: omnívoro arbóreo, OM: omnívoro, Pam: insectívoro con apariencia paseriforme sin percha para cazar del estrato arbustivo alto a medio, Pbh: insectívoro con apariencia paseriforme sin percha para cazar del estrato arbustivo bajo a herbáceo, RD: rapaz diurna, RN: rapaz nocturna, S: insectívoro que caza en el suelo o en la hojarasca, Tg: insectívoro grande a mediano de apariencia tiraniforme que caza desde perchas bajo el dosel arbóreo, Tp: insectívoro pequeño de apariencia tiraniforme que caza desde perchas bajo el dosel arbóreo.

Variables estructurales: DA: densidad de árboles (ind./ha), AB: área basal ( $\left.\mathrm{m}^{2} / \mathrm{ha}\right), \mathrm{C}$ : diámetro promedio de la copa arbórea $(\mathrm{m})$, AT: altura media del tronco $(\mathrm{m})$. Variables ambientales: Alt: altitud (msnm), HRS: humedad relativa en estación seca $(\%)$, HRL: humedad relativa en estación lluviosa (\%), TS: temperatura en estación seca $\left({ }^{\circ} \mathrm{C}\right)$, TL: temperatura en estación lluviosa $\left({ }^{\circ} \mathrm{C}\right)$. Valores $r$ : coeficiente de correlación no paramétrica de Spearman.

Status of protection based on NOM-059-Semarnat (Diario Oficial de la Federación 2010): In superscript A: endangered, PE: extinction risk, Pr: special protection. *Indicator species of physiographic region in Tabasco (IndVal $>0.70, \mathrm{p}<0.05$ ). (1) Former Coerebidae, (2) Former Cardinalidae. Superscript C-I, C-II and C-III represent CITES listed species (PNUMACMCM 2011).

Silvopastoral system: AL: trees in boundary-hedgerows, ADP: scattered trees in pastures.

G: Foraging guild: CA: aquatic hunter, Car: carrion-eating, Cro: bore-wood hunter or woodpecker, Gan: cattle associated hunter, Gfa: arboreal granivorous-frugivorous, Gft: terrestrial granivorous-frugivorous, N: nectarivorous, OA: arboreal omnivorous, OM: omnivorous, Pam: passeriform-like insectivorous without hunt perch from medium to high arboreal strata, Pbh: passeriform-like insectivorous without hunt perch of lower shrub or herbaceous strata, RD: diurnal raptor, RN: nocturnal raptor, S: insectivorous at soil or litter level hunter, Tg: medium or big size tyranniform-like insectivorous hunting below canopy, Tp: little insectivorous tyranniform-like hunting since perchs below canopy.

Structural variables: DA: trees density (ind./ha), AB: basal area $\left(\mathrm{m}^{2} / \mathrm{ha}\right), \mathrm{C}$ : mean tree-crown diameter $(\mathrm{m})$, AT: stem mean height (m). Environmental variables: Alt: altitude (mals), HRS: relative humidity in dry season (\%), HRL: relative humidity in rainy season $(\%)$, TS: dry season temperature $\left({ }^{\circ} \mathrm{C}\right)$, TL: rainy season temperature $\left({ }^{\circ} \mathrm{C}\right), r$ values: non parametric Spearman's correlation coefficient. 